Aus der Medizinischen Klinik mit Schwerpunkt Gastroenterologie, Hepatologie und Endokrinologie (Campus Mitte) der Medizinischen Fakultät Charité - Universitätsmedizin Berlin

\title{
DISSERTATION
}

\section{PROSPEKTIVER VERGLEICH VON POWER- DOPPLERSONOGRAPHIE UND ILEOKOLOSKOPIE IN DER AKTIVITÄTSBEWERTUNG BEI PATIENTEN MIT MORBUS CROHN}

zur Erlangung des akademischen Grades

Doctor medicinae (Dr. med.)

vorgelegt der Medizinischen Fakultät

Charité - Universitätsmedizin Berlin

von

Holger Neye

aus Potsdam 
Gutachter: 1. Priv.-Doz. Dr. med. habil. St. Rickes

2. Prof. Dr. med. J. Ricke

3. Prof. Dr. med. P. Malfertheiner

Datum der Promotion: 14.06.2009 


\section{Danksagung}

Die Medizin ist eine empirische Wissenschaft, ihre Urquelle sind menschliche Erfahrungen und Erlebnisse. Diese Eigenschaften der Medizin sind zugleich ihr größter Vorteil und Nachteil. Anders als beispielsweise die Mathematik, die auch ohne menschliche Schicksale existent bleibt, ist dies bei der Medizin nicht denkbar.

So sind klinische Lehrer, die mit ihren Erfahrungen ihre Schüler an die Grenze des heutigen Wissens bringen, für einen Erfolg von existenzieller Bedeutung.

Mein Lehrer im Ultraschall war Herr Prof. Wolfram Wermke, der mir nicht nur die Grundlagen vermittelte, wie ein Schallkopf mit der Hand geführt wird. Er lehrte auch die Philosophie, den Menschen, den dieser Schallkopf berührt, in allen medizinischen Entscheidungen im Mittelpunkt zu sehen. Für dieses Weitergeben eigener Erfahrungen gilt Herrn Prof. Wolfram Wermke mein lebenslanger Dank.

Neben den Lehrern brauchen Mediziner Freunde, deren ehrliche Kritik oft Leitpfad des eigenen Weges wird. Meinem langjährigen Kollegen, Freund und Mentor Herrn Chefarzt PD Dr. med. habil. Steffen Rickes gebührt großer Dank für seine Bereitschaft, meinen wissenschaftlichen Lebensweg mit seinem Rat und seiner Hilfe zu begleiten.

Auch bei Herrn Prof. Dr. med. Herbert Lochs, Klinikdirektor der Medizinischen Klinik mit Schwerpunkt Gastroenterologie, Hepatologie und Endokrinologie (Campus Mitte) der Charité, Universitätsmedizin Berlin, möchte ich mich für die Gewährung der notwendigen Rahmenbedingungen der vorliegenden Dissertation bedanken.

Stellvertretend für viele an dieser Stelle namentlich leider nicht Erwähnten möchte ich mich noch bei Schwester Marion Strelow bedanken, deren freundliche und zuvorkommende Art nicht nur den Patienten ein Licht im Klinikalltag war.

Abschließend danke ich allen meinen Patientinnen und Patienten, deren Vertrauen in meine Arbeit, Grundlage und Zielsetzung meiner beruflichen Tätigkeit ist und bleibt. 


\section{Inhaltsverzeichnis}

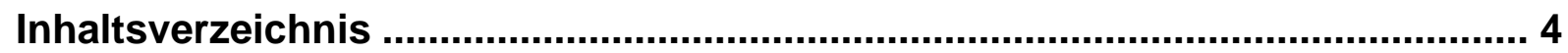

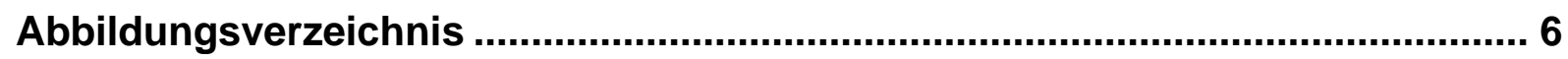

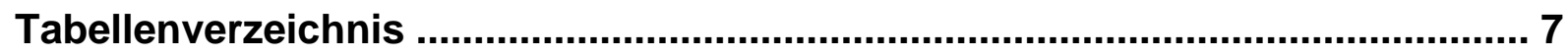

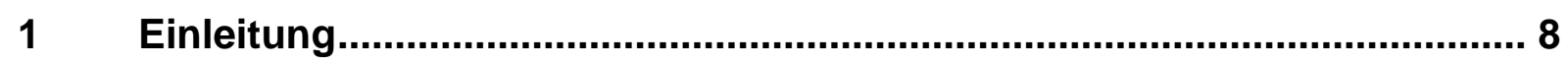

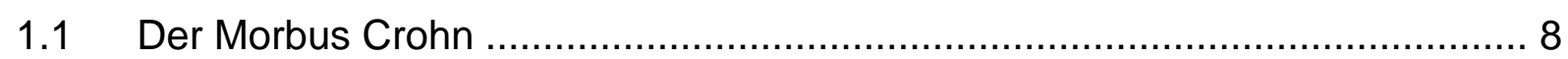

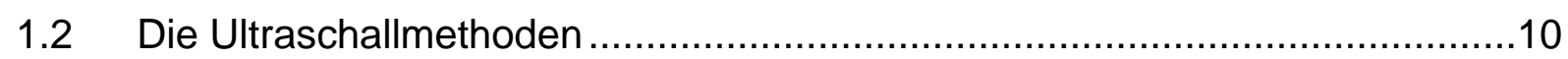

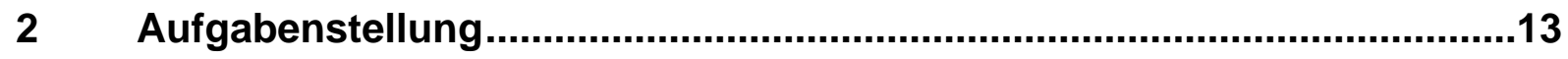

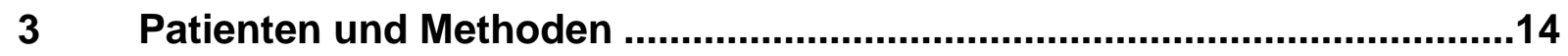

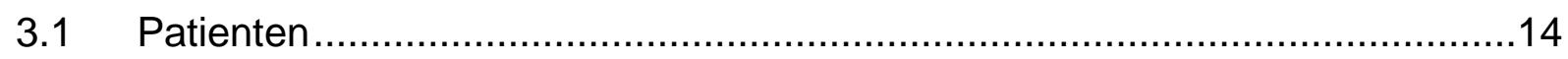

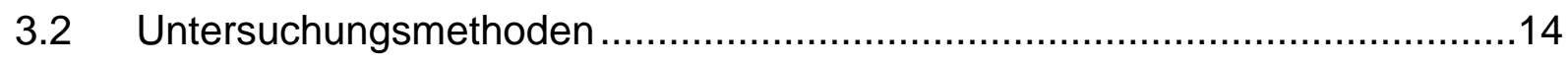

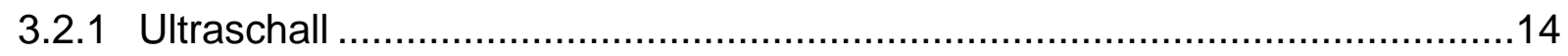

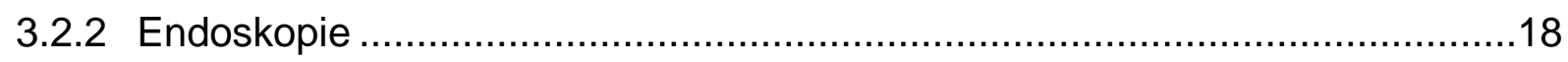

3.2.3 Der klinische Aktivitätsindex Crohn's Disease Activity Index (CDAI) ….........19

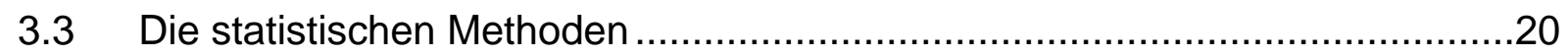

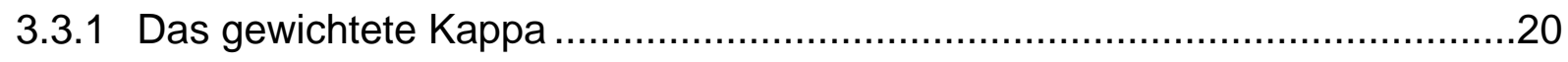

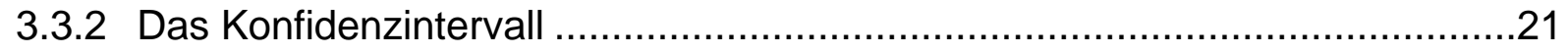

3.3.3 Sensitivität, Spezifität, positiver und negativer prädiktiver Wert .....................22

$4 \quad$ Ergebnisse

4.1 Power-Dopplersonographie und Ileokoloskopie in der Aktivitätsbewertung....24 
4.2 Sensitivität und Spezifität der Power-Dopplersonographie in der Beurteilung der Ausdehnung der Entzündung bei Patienten mit einem Morbus Crohn .....29

4.3 Die Ergebnisse des Vergleichs mit dem klinischen Aktivitätsindex CDAl .......30

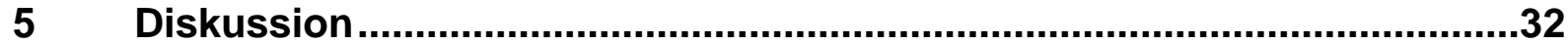

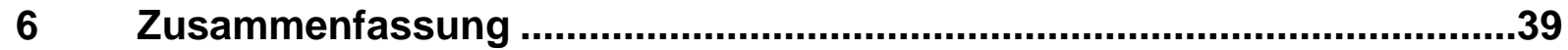

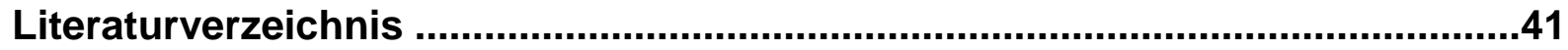

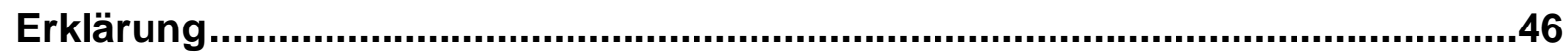

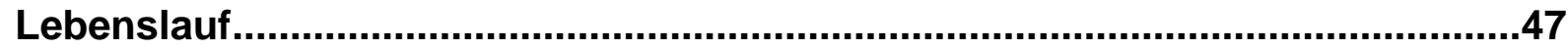




\section{Abbildungsverzeichnis}

Abbildung 1: Verdickte Sigmaschlinge untersucht mit der Power-

Dopplersonographie, der Abschnitt mit der stärksten Vaskularisation wird mit dem Sektorschallkopf selektiert.

Abbildung 2: Spektrum einer Arterie und einer Vene im PW-Doppler: damit Blutfluss, keine Bewegungsartefakte im Power-Mode

Abbildung 3: Vereinzelte Gefäße in der echogenen Submucosa des Colon

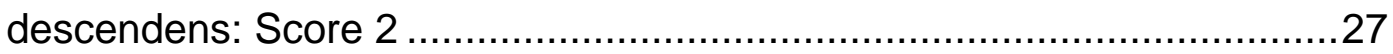

Abbildung 4: Gefäße, die sich in der verdickten Wand des terminalen lleums aufzweigen: Score 3

Abbildung 5: Hypervaskularisation mit Nachweis zuführender Gefäße, die die Wand des Colon ascendens penetrieren: Score 4. Das Flusssignal ist kräftig, der Farbdoppler detektiert die Richtung. 


\section{Tabellenverzeichnis}

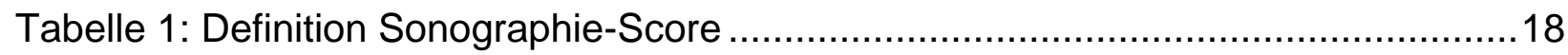

Tabelle 2: Mögliche Score-Differenzen............................................................21

Tabelle 3: Kappa- Koeffizient und Konkordanz (Landis und Koch)..........................21

Tabelle 4: Ergebnisse der Power-Dopplersonographie im Vergleich zur

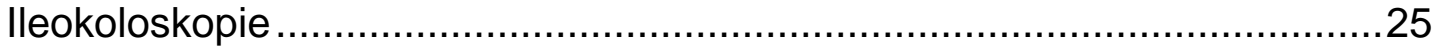

Tabelle 5: Score-Resultate von Power-Dopplersonographie und Ileokoloskopie ..........26

Tabelle 6: Vergleich der sonographischen Score-Summe mit dem CDAI ....................31 


\section{Einleitung}

\subsection{Der Morbus Crohn}

Patienten mit einem Morbus Crohn leiden an einer Erkrankung, deren charakteristische Merkmale der unbestimmte Verlauf, die unbestimmte Ausdehnung und die unbestimmte Aktivität sind.

Der unbestimmte Verlauf der Erkrankung umfasst Perioden, in denen der Morbus Crohn aktiv ist, die durch inaktive Phasen abgewechselt werden, in welchen die Patienten ein fast normales Leben führen können. Der Zeitpunkt und die Dauer der aktiven Krankheitsphase sind den behandelnden Ärzten und den betroffenen Patienten vor ihrem Auftreten nicht bekannt. Anders als bei anderen chronischen Erkrankungen lässt sich weder dem einzelnen Patienten noch einer Gruppe von Patienten eine wiederkehrende Abfolge der Erkrankung, eine Periodik, zuordnen [Sonnenberg et al. 1982,Schölmerich 2003].

Die unbestimmte Ausdehnung bedeutet, die Orte einer Entzündung in einer vorangegangenen Krankheitsphase variieren häufig gegenüber der aktuellen Krankheitsphase, zuvor gesunde oder nur gering entzündlich veränderte Abschnitte des Verdauungstraktes können aktuell eine schwere Entzündung aufweisen und umgekehrt [Maconi et al. 2006].

Die unbestimmte Aktivität bedeutet, das Ausmaß der Entzündung an einem definierten Abschnitt des Gastrointestinaltrakts kann sich in den verschiedenen Krankheitsphasen verändern, so kann der Darm an diesem Ort aktuell gesund sein, nur geringgradig entzündlich verändert, mäßiggradig oder hochgradig entzündet [Meckler et al. 1991].

Erschwerend kommt mit jedem weiteren Krankheitsschub die Problematik von chronisch-fibrosierenden Veränderungen hinzu. Der Darm verliert oft relativ kurzstreckig seine Elastizität, Verengungen resultieren daraus. Der Darmbrei staut sich vor diesen Stenosen, eine (Sub-)ileus-Symptomatik ist die Folge. Diese Stenosen zeigen keine Entzündungsaktivität, sind sie doch Folge von fibrotischem Umbau, der nach den eigentlichen Entzündungen entstanden ist [Maconi et al. 1996; Miao et al. 2002]. 
Oft verbleibt hier als ultima ratio nur eine chirurgische Intervention, die sich auf einen minimal-invasiven Eingriff beschränken sollte. Wenn die Intervention zu spät entschieden wird, riskiert der Patient den Verlust längerer Abschnitte des Darmes bei Notoperationen [Fraquelli et al. 2005, Mackalski et al. 2006].

Stenosen, die durch eine akute Entzündung verursacht werden, zeigen häufig eine ähnliche Symptomatik. Im Gegensatz zu den fibrotischen Veränderungen sollte hier jedoch keine chirurgische Intervention erfolgen, eine Optimierung der anti-inflammatorischen Therapie ist anzustreben. Der Erfolg einer Therapie ist abhängig von der richtigen Wahl des Medikamentes, seiner Applikationsart (zum Beispiel Klysma, Kapsel, intravenöse Gabe) und seiner Galenik. Applikation und Galenik sind durch den behandelnden Arzt zu entscheiden. Diese Entscheidung hängt von der Lokalisation, der Ausdehnung und der Aktivität der Entzündung ab [Ambrosini et al. 2007].

Dieses Wissen kann der Arzt nur durch bildgebende Verfahren erlangen, da Laborparameter oder vom Patienten ausgefüllte Fragebögen, die sein Befinden widerspiegeln, nur den allgemeinen, d.h. systemischen Krankheitszustand beschreiben können. Der Zustand der einzelnen Darmabschnitte, der zum Beispiel zur Entscheidung für oder gegen eine Operation wichtig ist, bleibt im Verborgenen [Heyne et al. 2002, Hirche et al. 2002].

Für den Bereich des Colons und des terminalen Ileums besitzt hierfür die lleokoloskopie eine hervorragende Bedeutung, gestattet sie doch eine Beschreibung vom Ort, der Ausdehnung und der Aktivität der Entzündung. Ihr Nachteil ist ihre Invasivität, durch die Möglichkeit von Komplikationen und insbesondere von Schmerzen während der Untersuchung, die nicht zuletzt durch die Entzündung selbst aggraviert werden, kann sie zur Aktivitätsbestimmung nicht beliebig häufig durchgeführt werden. Allerdings sind ein sicherer Ausschluss eines kolorektalen Karzinoms und eine histologische Sicherung nur durch die Ileokoloskopie möglich, in absehbarer Zukunft ist sie für diese Indikationen durch andere bildgebende Verfahren nicht ersetzbar [Schölmerich 2003].

Zur Bestimmung der Ausdehnung und der Aktivität einer Entzündung sind jedoch für Verlaufskontrollen andere, nicht-invasive, Verfahren anzustreben. Durch die Einführung neuer Medikamente in der Behandlung des Morbus Crohns steigt die Bedeutung von häufigeren Verlaufskontrollen, da so befundabhängig über die Fortführung bzw. Dosis- 
änderung der Medikation entschieden werden kann. Auch kann dadurch eine teure Therapieform gegenüber dem Versicherungsträger besser gerechtfertigt werden, oder im umgekehrten Fall eine nicht nur teure, sondern für den Patienten auch mit Nebenwirkungen behaftete medikamentöse Behandlung in ihrer Dosis rechtzeitiger reduziert bzw. sogar beendet werden [Ambrosini et al. 2007].

Für häufigere Verlaufskontrollen bei Patienten mit Morbus Crohn wäre eine Bildgebung wünschenswert, die nicht-invasiv ist und auf Grund ihres Nebenwirkungsprofils nahezu beliebig oft auch bei jüngeren Patienten wiederholt werden kann. Der transabdominelle Ultraschall als ein nicht-invasives Verfahren, das nicht mit einer Röntgenbelastung assoziiert ist und keine ernsthaften Nebenwirkungen hat, kann diese Voraussetzungen erfüllen.

\subsection{Die Ultraschallmethoden}

Die Entwicklung des bildgebenden Ultraschalls begann mit dem A-Mode (von A: amplitude), entlang einer Geraden wird die Intensität des rückkehrenden Schallstrahls als Amplitude dargestellt. Hierbei handelt es sich um ein eindimensionales Verfahren in seiner Erzeugung (x-Achse), in der Darstellung wird jedoch schon eine zweite Dimension, die dem Verfahren den Namen gab, die Amplitude (als y-Achse) genutzt.

Die Idee, diese Amplitude beim B-Mode (B = brightness) als Punkt verschiedener Helligkeit dem menschlichen Auge zu demonstrieren, ermöglichte eine zweidimensionale Darstellung von normalen und pathologisch veränderten Organen. Der Erfolg des Ultraschalls war mit der Einführung des B-Mode verbunden, durch die Darstellung in zwei Dimensionen entstand ein echtes Schnittbildverfahren.

Beim transabdominellen Ultraschall kann der Untersucher frei mit seiner Hand die $\mathrm{x}$ und $y$ - Achse in den menschlichen Körper legen. Das ist ein großer Vorteil in der Festlegung der Maximalausdehnung von dreidimensionalen Strukturen, beispielsweise einer Raumforderung. Stellen wir uns hierzu ein Ellipsoid, ein Gebilde wie ein Rugby-Ball, frei schwebend im Raum vor: Der Untersucher kann den Ultraschallkopf so wenden, bis die eigentliche Raumachse der Raumforderung direkt im zweidimensionalen Schnittbild liegt. Mit CT oder MRT gelingt dies nicht, da diese Verfahren durch die Geräteposition an drei definierte Ebenen, der Sagittal-, Transversal- und Koronarebene, gebunden sind. Liegt das zu untersuchende Ellipsoid im Raum schief zu allen drei Grundebenen, 
ist die eigentliche zweidimensionale Struktur, die Ellipse, für den Betrachter so verzerrt kaum erkennbar.

Diese Vorteile des Ultraschalls werden mit dem Nachteil einer oft schwer zugänglichen Vermittelbarkeit der Bilder erkauft, da der Untersucher mit seiner Hand willkürlich aus unendlich vielen Winkeln der drei Grundebenen des Raumes wählen kann. Anders als bei CT oder MRT sind nachträgliche Befundungen von bereits angefertigten Bildern durch einen Untersucher mit größerer Erfahrung meist nicht sinnvoll, und auch die chirurgischen Fächer können im Operationssaal mit angefertigten Ultraschallbildern ihre OP-Planung nicht vergleichbar gut umsetzen.

Von allen abdominellen Organen besitzt der Darm in Bezug auf den dreidimensionalen Verlauf die größte Variabilität. Die bildgebenden Verfahren, die an die drei definierten Ebenen gebunden sind, projizieren in ihren Bildern die Wanddicke in Abhängigkeit vom Anschnittwinkel sehr unterschiedlich, je schräger der Winkel umso größer die Wanddicke. Nur wenn die Schnittebene orthogonal zur Darmwand liegt, wird ihre Dicke korrekt abgebildet. Im Gegensatz zu CT und MRT kann der erfahrene Untersucher mit dem Ultraschallkopf aus den unendlichen vielen möglichen Schnittwinkeln den orthogonalen schnell selbst auswählen. Auch kann er den interindividuellen sehr unterschiedlichen Verlauf des Darmes, beispielsweise vom Colon transversum, durch die Freiheit seiner Handbewegungen nachvollziehen.

Diese Vorteile des Ultraschalls für Patienten mit Morbus Crohn zu nutzen, wurde bereits vor der Einführung der Dopplerverfahren nur unter Anwendung des B-Mode versucht [Holt et al. 1979, Wellmann et al. 1980]. Spätere Untersuchungen zeigten allerdings, dass eine Unterscheidung rein entzündlicher Wandverdickungen von chronischfibrotischen mit Hilfe des Ausmessens der Wandverdickung im B-Bild zu falschen Aussagen führen kann [Maconi et al. 1996]. Die Aussagekraft der Darmwanddicke bezüglich der Aktivität einer Entzündung ist vom Alter der Patienten mit einem Morbus Crohn abhängig. So konnten Haber et al. eine sehr gute Korrelation zwischen der Wanddicke des Darmes und der Aktivität seiner Entzündung zeigen, allerdings waren es ausschließlich pädiatrische Patienten, bei denen noch nicht rezidivierende Entzündungen des Darmes diesen fibrosieren ließen. 
Ältere Arbeiten der Angiographie [Bousen et al. 1966, Lunderquist 1967] wiesen bereits auf die Bedeutung der Gefäße innerhalb der Darmwand für die Aktivitätsbeurteilung einer entzündlichen Reaktion hin. Obwohl seit Anfang der 80-iger Jahre mit Hilfe des Farbdopplers auch Gefäße mit ihrem Fluss sonographisch dargestellt werden konnten, waren die Gefäße innerhalb der Darmwand auch bei Entzündung zu klein und die Geschwindigkeit des Flusses zu gering für ihre Detektion [Sonnenberg et al. 1982].

Erst durch die Einführung neuer Dopplerverfahren wie der Powerdopplersonographie [Bude et al. 1994, Newman et al. 1994, Rubin et al. 1994, Martinoli et al. 1998], die es vermögen, langsame Blutflüsse in sehr kleinen Gefäßen zu detektieren, stieg wieder die Hoffnung, den Ultraschall für die Bestimmung der Aktivität einzelner Darmabschnitte zu nutzen [Heyne et al. 2002]. 


\section{Aufgabenstellung}

Die meisten gastroenterologischen Zentren nutzen heute die endoskopischen Verfahren zur Bestimmung der Aktivität von einzelnen Darmabschnitten bei Patienten mit Morbus Crohn. Falls die Sonographie helfen soll, Verlaufskontrollen zwischen den endoskopischen Kontrollen zu ermöglichen, ist die Frage nach einem Vergleich beider diagnostischer Methoden in der Beurteilung der Aktivität von einzelnen Darmsegmenten von essentieller Bedeutung.

Es ist zu klären, ob die Einschätzung der entzündlichen Aktivität durch die Sonographie unter Einbeziehung der neuen Dopplermethoden im Vergleich mit der Ileokoloskopie zu ähnlichen oder zu divergenten Ergebnissen führt.

Hierzu ist es notwendig, diagnostische Kriterien für die Power-Dopplersonographie zu erarbeiten. Ziel dieser prospektiven Studie ist die Evaluierung der PowerDopplersonographie in der Bestimmung der Aktivität differenter Darmsegmente im Vergleich mit der lleokoloskopie bei Patienten mit Morbus Crohn. Durch die vorliegende Arbeit soll ein Beitrag geleistet werden, um Patienten mit einem Morbus Crohn zukünftig schonend, aussagesicher und schnell zu diagnostizieren.

Für die Entscheidung zu weiteren therapeutischen Maßnahmen bzw. zur Entscheidung über Dosisänderungen einer medikamentösen Therapie bei Patienten mit einem Morbus Crohn sind die Einschätzung der Aktivität einzelner Darmsegmente sowie die Beurteilung der Ausdehnung der Erkrankung zu diagnostizieren. Die vorliegende Arbeit soll dabei folgende Fragen klären:

1. Führt die Einschätzung der entzündlichen Aktivität einzelner Darmsegmente durch die Sonographie unter Einbeziehung der neuen Dopplermethoden im Vergleich mit der lleokoloskopie zu konkordanten oder zu divergenten Ergebnissen?

2. Kann die Sonographie die Ausdehnung der Entzündung bei Patienten mit einem Morbus Crohn korrekt einschätzen? 


\section{Patienten und Methoden}

\subsection{Patienten}

In diese prospektive Studie wurden 31 konsekutive Patienten (18 Frauen, 13 Männer) der Medizinischen Klinik mit Schwerpunkt Gastroenterologie, Hepatologie und Endokrinologie des Universitätsklinikums Charité Berlin (Campus Mitte) mit einem bereits vor dem Beginn der Studie gesicherten Morbus Crohn eingeschlossen. Das mittlere Lebensalter betrug 34,8 Jahre, wobei der jüngste Patient 18 Jahre war und die älteste Patientin ein Lebensalter von 56 Jahren hatte. Die mittlere Krankheitsdauer seit Stellung der Diagnose eines Morbus Crohns bis zum Studieneinschluss betrug 7,2 Jahre.

Alle Patienten gaben nach ausführlicher Aufklärung ihr Einverständnis zur Teilnahme an dieser Studie. Das Protokoll wurde zuvor von der Ethikkommission der Charité Berlin genehmigt.

Alle 31 Patienten wurden prospektiv mit B-Mode und Power-Dopplersonographie sowie mit der Ileokoloskopie untersucht. Bei 4 Patienten lag ein Zustand nach einer Darmresektion vor. Bei allen diesen 4 Patienten wurden das Coecum und das terminale lleum reseziert. Bei einem der 4 Patienten umfasste die Resektion auch das Colon ascendens, bei einem anderen wurde das Ileum längerstreckig entfernt.

\subsection{Untersuchungsmethoden}

\subsubsection{Ultraschall}

Sämtliche sonographischen Untersuchungen führte Holger Neye durch. Der Untersucher war zu den klinischen Daten und den Ergebnissen der übrigen Untersuchungsverfahren verblindet.

Die Sonographie erfolgte binnen 3 Tagen vor der Endoskopie mit hochauflösenden Schallköpfen (dynamischer Linearscanner 5-12 MHz und dynamischer Sektorscanner 4-7 MHz, HDI 5000, Philips Ultrasound). Definierte Ultraschallparameter (Darmwanddicke, Vaskularisationsmuster) wurden bei jedem Patienten separat in einem standardisierten Protokoll für 6 differente Darmsegmente bestimmt. Diese Darmsegmente waren das Sigma, das Colon descendens, das Colon transversum, das Colon ascendens, das Coecum sowie das terminale Ileum. Die sonographische Zuordnung der einzelnen 
Darmsegmente erfolgte in der Untersuchung mit dem Sektorscanner, da nur er den hierzu notwendigen Überblick ermöglicht. Vor der jeweiligen Zuordnung wurde der Darm in seiner Kontinuität vom terminalen Ileum bis zum Sigma dargestellt, um beispielsweise Verwechslungen eines sehr kaudal verlaufenden Colon transversum mit einem rechtslateral elongierten Sigma vermeiden zu können. Jedes einzelne Darmsegment musste Bedingungen erfüllen, die vor dem Beginn der Studie festgelegt wurden. Das terminale Ileum musste linkslateral in den übrigen Dünndarm übergehen, rechtslateral wurde die Darstellung der Bauhinschen Klappe bzw. im Falle eines neoterminalen Ileums die Darstellung der Anastomose gefordert. Die Bauhinsche Klappe war zugleich die trennende Struktur zwischen einem nach kaudal blind auslaufenden Colonabschnitt, dem Coecum, und dem Anfang des Colon ascendens. Besondere Bedeutung besitzt hierbei die korrekte Lagebestimmung der rechten sowie linken CoIonflexur. Beide Flexuren erfordern in besonderem Maß vom Untersucher eine dreidimensionale Vorstellung, da die Lagebeziehung vom Colon transversum zum CoIon ascendens bzw. zum Colon descendens streng laterolateral oder streng ventrodorsal sein und auch alle Nuancen zwischen diesen Extremen auftreten. Als Flexur wurde der Ort mit der stärksten Änderung der longitudinalen Darmachse definiert, die rechte Colonflexur trennt hierbei das Ende des Colon ascendens vom Anfang des Colon transversum, dessen Ende in der linken Flexur zugleich der Anfang des Colon descendens ist. Das Colon descendens ist sehr linkslateral in seinem Verlauf verfolgbar, wobei es oft einen kraniokaudal geraden Verlauf zeigt. Sein Ende wird von einem Punkt unterhalb des Nabels definiert, bei dem die longitudinale Darmachse die erste größere Abweichung zeigt, denn hier beginnt das Sigma. Dessen Ende wird durch die Darstellung der Harnblase sichtbar. Transvesikal zeigt sich der L-förmige Verlauf des Rectums, dessen Beginn das Ende des Sigmas definiert. Die Beurteilung des Rectums wurde in das Studienprotokoll nicht einbezogen. Für eine korrekte Beurteilung der Entzündungsaktivität des Rectums ist es sinnvoll, eine Untersuchung mit einem Linearscanner vom Perineum aus und eine Untersuchung mit einem transanal applizierbaren Schallkopf zu kombinieren. Diese Kombination ist in ihrer Durchführung deutlich vom transabdominellen Ultraschall different, eine Schwächung der statistischen Aussagekraft wäre die Folge gewesen. Aus diesem Grund wurde das Rectum bereits in der Vorbereitung der Studie nicht in ihr Protokoll aufgenommen. 
Zuerst erfolgte eine sonographische Untersuchung der beschriebenen 6 Darmsegmente mit dem Sektorschallkopf unter den Bedingungen des B-Modes, um eine Verdickung der Darmwand zu detektieren. Die verdickte Darmschlinge wurde dann mit der PowerDopplersonographie untersucht, um den Abschnitt mit der stärksten Vaskularisation des betreffenden Segments zu selektieren (Abbildung 1).

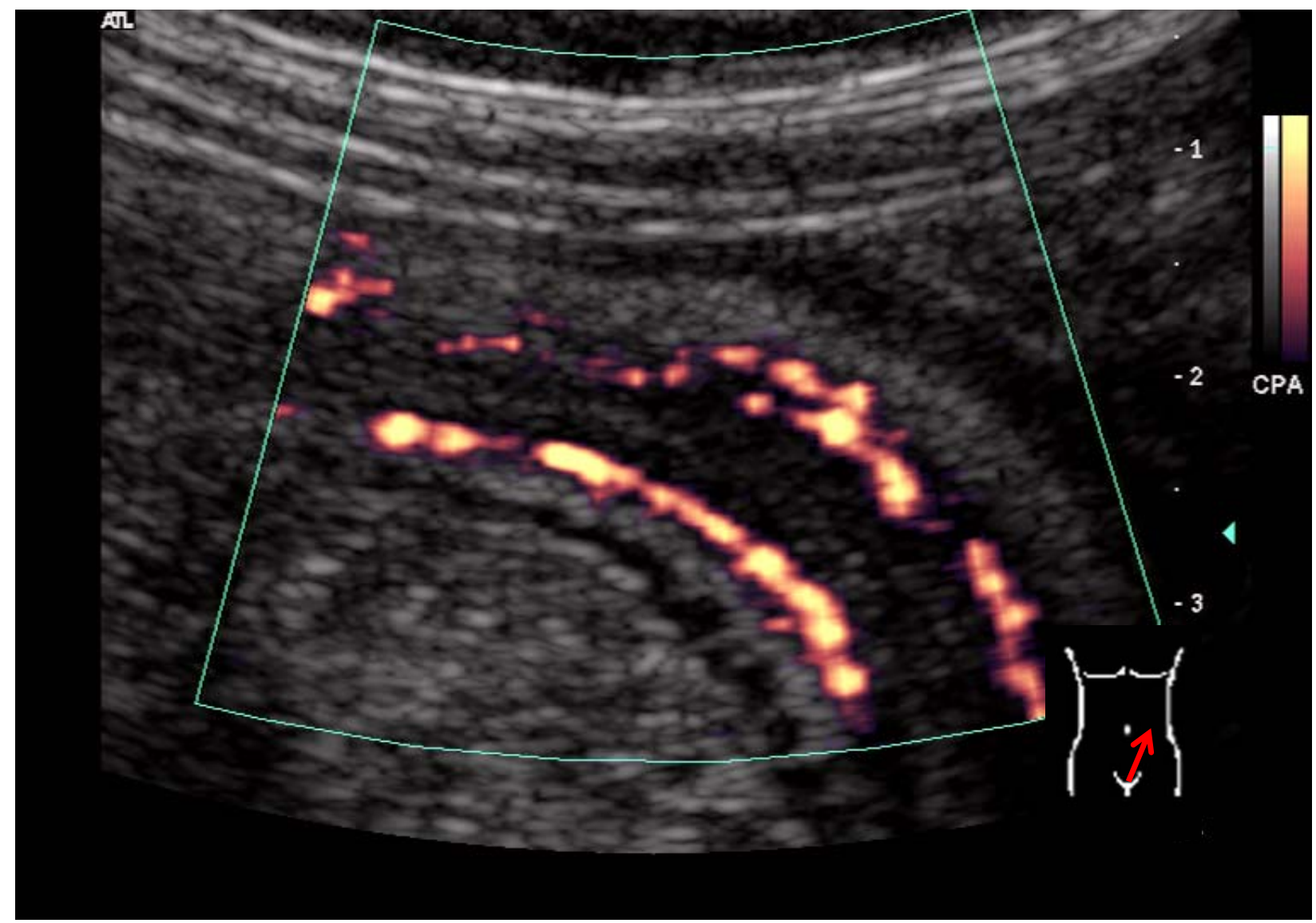

Abbildung 1: Verdickte Sigmaschlinge untersucht mit der Power-Dopplersonographie, der Abschnitt mit der stärksten Vaskularisation wird mit dem Sektorschallkopf selektiert. 
Zur sicheren Unterscheidung zwischen echten Gefäßsignalen und Bewegungsartefakten wurde der PW-Doppler verwendet (Abbildung 2). Dann wurde mit dem Linearschallkopf das Maximum der Wandverdickung in diesem Segment durch Messung der Lumen-Serosa-Distanz bestimmt. Nachfolgend erfolgte die Bewertung des Vaskularisationsmusters, das aus der Anzahl der nachweisbaren Gefäße pro Quadratzentimeter bestimmt wurde.

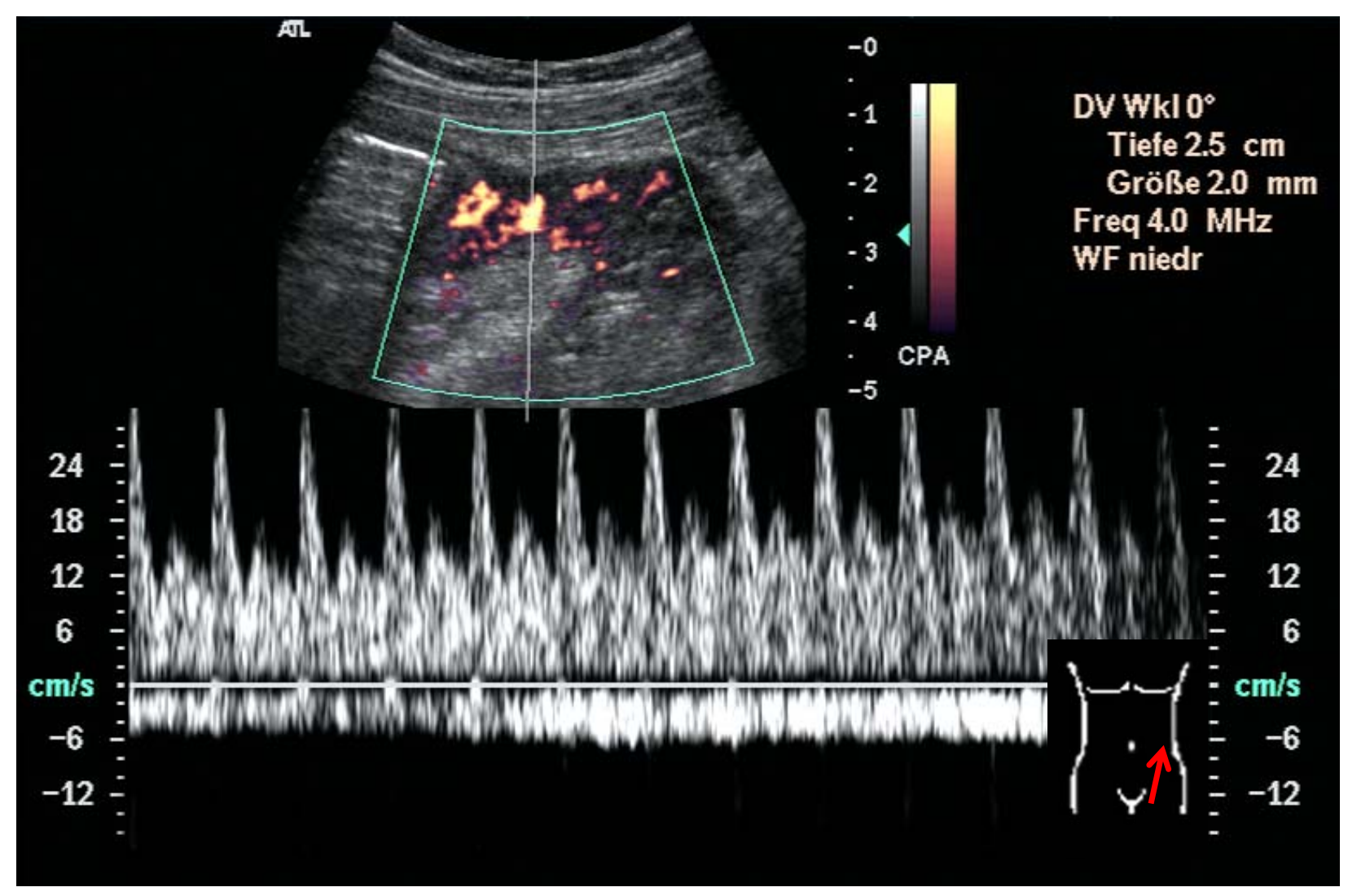

Abbildung 2: Spektrum einer Arterie und einer Vene im PW-Doppler: damit Blutfluss, keine Bewegungsartefakte im Power-Mode

Der B-Mode (maximale Wandverdickung des betreffenden Darmsegments) sowie die Ergebnisse der Power-Dopplersonographie (Vaskularisationsmuster) determinierten den sonographischen Score der Entzündungsaktivität (Tabelle 1). 
Tabelle 1: Definition Sonographie-Score

\begin{tabular}{|c|c|c|c|}
\hline 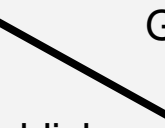 & keine Gefäße $/ \mathrm{cm}^{2}$ & 1-2 Gefäße/cm² & > 2 Gefäße/cm² \\
\hline$<5 \mathrm{~mm}$ & 1 & 2 & 3 \\
\hline$\leq 7 \mathrm{~mm}$ & 2 & 3 & 4 \\
\hline$>7 \mathrm{~mm}$ & 2 & 3 & 4 \\
\hline
\end{tabular}

Wie aus der Tabelle 1 ersichtlich ist, erfolgte eine Einteilung der sonographischen Entzündungsaktivität in 4 Scores, wobei der Score 1 dem fehlenden Nachweis einer Entzündung entspricht, Score 2 einer geringen, Score 3 einer mäßigen und Score 4 einer hohen Entzündungsaktivität des Morbus Crohn im betreffenden Darmsegment.

\subsubsection{Endoskopie}

Sämtliche Ileokoloskopien wurden ebenfalls von erfahrenen Untersuchern durchgeführt, die zu den klinischen Daten und den Ergebnissen der übrigen diagnostischen Verfahren verblindet waren.

Die endoskopischen Parameter (Muster und Ausdehnung typischer Läsionen) wurden in einem standardisierten Protokoll für die identischen 6 Darmsegmente dokumentiert. Diese Darmsegmente waren wie bei der zuvor erfolgten Darmsonographie das Sigma, das Colon descendens, das Colon transversum, das Colon ascendens, das Coecum sowie das terminale lleum. Auch mit der lleokoloskopie erfolgte eine Einteilung der Entzündungsaktivität in 4 Scores, wobei der Score 1 dem fehlenden Nachweis von Schleimhautläsionen entspricht, beim Score 2 einzelne Aphthen nachweisbar sind, bei Score 3 Aphthen und Ulzerationen bei weniger als 50\% der Schleimhaut sichtbar sind und bei Score 4 weisen mindestens 50\% oder mehr der Schleimhaut Aphthen und Ulzerationen auf. 


\subsubsection{Der klinische Aktivitätsindex Crohn's Disease Activity Index (CDAI)}

Zur Bestimmung der klinischen Krankheitsaktivität sind mehrere Indizes entwickelt worden. In der überwiegenden Zahl der publizierten Studien zum Morbus Crohn und auch in der S3-Leitlinie „Diagnostik und Therapie des Morbus Crohn“ der Deutschen Gesellschaft für Verdauungs- und Stoffwechselkrankheiten in der aktuellen Fassung vom Oktober 2008 [Hoffmann et al. 2008] wird hierzu der Crohn's Disease Activity Index [Best et al. 1976] verwendet. In diesen Index gehen mit einer Wichtung, die durch einen Multiplikationsfakor vermittelt wird, klinische und paraklinische Angaben und Daten als ein Summenwert ein. Hierbei werden anamnestische Angaben verwendet wie Fragen zum Stuhlgang, dem Grad der empfundenen Bauchschmerzen, der Beeinträchtigung des Allgemeinbefindens sowie der Frage nach begleitenden Gelenkschmerzen. Desweiteren werden zur Bestimmung des CDAl Angaben der klinischen Untersuchung genutzt wie die Existenz von Symptomen (Erythema nodosum, Iritis, Uveits, Analfissur, Fisteln) oder die Ausprägung einer palpablen Resistenz im Abdomen. Auch Meßgrößen wie der Hämatokritwert und das Körpergewicht fließen in seine Berechnung ein.

Bei sämtlichen Patienten der vorliegenden Studie wurde der CDAI ermittelt. Dabei wurde ein Wert $\leq 150$ als ein Morbus Crohn in Remission gewertet, eine aktive Erkrankung lag vor, wenn der CDAI >150 war. Diese Einteilung basiert auf der S3-Leitlinie „Diagnostik und Therapie des Morbus Crohn" der Deutschen Gesellschaft für Verdauungsund Stoffwechselkrankheiten [Hoffmann et al. 2008].

Um den CDAl eines Patienten mit seinen sonographischen Ergebnissen vergleichen zu können, ist eine Summenbildung notwendig, da jeder Patient getrennte Bewertungen von 6 Darmsegmenten erhält. Bei völliger Gesundheit erreicht ein Patient eine minimale Score-Summe von 6, da jedes Segment den Score 1 aufweist. Der maximal mögliche Wert der Score-Summe würde sich ergeben, wenn jedes Segment den Score 4 erhält, woraus eine Scoresumme von 24 resultieren würde.

Als Grenze zwischen einer Remission und einer aktiven Entzündung wurde eine Scoresumme von einem Wert $\leq 9$ festgelegt. Dies bedeutet, eine aktive Entzündung liegt vor, wenn mehr als drei Segmente nur gering entzündet sind und damit den Score 2 erhalten bzw. wenn weniger Segmente betroffen, jedoch eine höhere Ausprägung der Entzündung als den Score 2 besitzen. 


\subsection{Die statistischen Methoden}

Als erste Frage der Studie muss beantwortet werden, ob die Einschätzung der entzündlichen Aktivität einzelner Darmsegmente durch die Sonographie unter Einbeziehung der neuen Dopplermethoden konkordante oder divergente Ergebnisse im Vergleich zur Ileokoloskopie zeigt.

Dies betrifft nicht nur die Frage, ob das betreffende Darmsegment entzündet ist oder nicht. Auch eine Beurteilung des Grades der Aktivität in einer Graduierung von 4 möglichen Zuständen ist hierfür notwendig. Dabei wird bei jedem Patienten jedes der 6 Darmsegmente durch beide Verfahren beurteilt, der Sonographie sowie der Ileokoloskopie, wobei 4 Kategorien möglich sind. Diese Kategorien liegen in einer Ordinalskalierung vor, da sie eine zunehmende Aktivität der Entzündung widerspiegeln. Als Maß der Übereinstimmung zweier Verfahren bezüglich mehrerer Kategorien eignet sich als bester Konkordanzindex das gewichtete Kappa [Altman 1991, Sachs 1999].

Die gewichteten Kappa-Werte sowie ihre Konfidenzintervalle wurden mit StatXact, Version 5.0.3 (Cytel software) berechnet.

\subsubsection{Das gewichtete Kappa}

Das gewichtete Kappa bewertet den Grad an Übereinstimmung zweier Untersuchungsverfahren, wobei beide Verfahren mehrere Kategorien der Bewertung besitzen, in der vorliegenden Studie sind es vier.

Zur Veranschaulichung kann man sich eine Tafel von 4 x 4 Feldern (siehe Tabelle 2) vorstellen, wobei die Spalten von den 4 möglichen endoskopischen und die Zeilen von den 4 möglichen sonographischen Scores von 1 bis 4 gebildet werden. Die Hauptdiagonale weist den höchsten Grad an Übereinstimmung auf, denn hier erfolgt durch beide Verfahren eine identische Zuordnung. Würden alle Resultate zu diesen 4 Feldern zugeordnet werden können, würde ein gewichtetes Kappa von 1 resultieren. Der umgekehrte Fall würde entstehen, wenn nur die beiden äußeren Felder mit der ScoreDifferenz von 3 belegt würden, dann resultiert ein gewichtetes Kappa von 0, es liegt keine Übereinstimmung zwischen den beiden Verfahren vor [Graham und Jackson 1993, Munoz et al. 1997, Chmura et al. 2002, Kundel et al. 2003, Sim et al. 2005]. 
Tabelle 2: Mögliche Score-Differenzen

\begin{tabular}{|l|c|c|c|c|}
\hline & \multicolumn{4}{|c|}{ lleokoloskopie } \\
\hline Sonographie & Score : 1 & Score : 2 & Score : 3 & Score: 4 \\
\hline Score : 1 & 0 & 1 & 2 & 3 \\
\hline Score : 2 & 1 & 0 & 1 & 2 \\
\hline Score : 3 & 2 & 1 & 0 & 1 \\
\hline Score : 4 & 3 & 2 & 1 & 0 \\
\hline
\end{tabular}

Auch zwischen den beiden Extrempunkten, einer völlig fehlenden Übereinstimmung mit einem gewichteten Kappa von 0 und einer völligen Übereinstimmung mit einem gewichteten Kappa von 1 ist der Grad der Konkordanz durch die Einteilung nach Landis und Koch festgelegt (1977), die in der Tabelle 3 dargestellt wird.

Tabelle 3: Kappa- Koeffizient und Konkordanz (Landis und Koch)

\begin{tabular}{|l|c|c|c|c|c|c|}
\hline $\begin{array}{l}\text { gewichteter }(\kappa) \\
\text { Koeffizient }\end{array}$ & 0 & $<0.21$ & $0.21-0.40$ & $0.41-0.60$ & $0.61-0.80$ & $>0.81$ \\
\hline $\begin{array}{l}\text { Grad der } \\
\text { Konkordanz }\end{array}$ & keine & $\begin{array}{c}\text { sehr } \\
\text { schwach }\end{array}$ & schwach & moderat & stark & sehr stark \\
\hline
\end{tabular}

\subsubsection{Das Konfidenzintervall}

Der Begriff "Confidence interval" wurde 1950 von J. Neyman und E.S. Pearson eingeführt [Sachs 1999].

Aus einer endlichen Stichprobenmenge will man auf die Eigenschaften einer (zumindest mathematisch) unendlichen Menge von möglichen "Wahrheiten", der Grundgesamtheit, schließen.

Man wählt üblicherweise ein 95\%-Konfidenzintervall (in deutscher Literatur auch Synonym: Vertrauensbereich), es bedeutet dass bei häufiger Anwendung des Ver- 
fahrens die berechneten Vertrauensbereiche in etwa 95\% der Fälle den Parameter überdecken und in nur 5\% nicht erfassen. An sich ist 95\% willkürlich gewählt, man könnte auch 70, 80, 90 oder 99\% nehmen. Je höher die Prozentzahl jedoch ist, umso weiter wird das Konfidenzintervall, so hat sich 95\% als ein guter Kompromiss eingebürgert, der auch bei der vorliegenden Studie gewählt wurde. Die Formel für das Konfidenzintervall ist vom gewählten Parameter abhängig, in der vorliegenden Studie vom gewichteten Kappa-Wert. Mit 95\% Wahrscheinlichkeit liegt der betreffende Parameter (hier das gewichtete Kappa) innerhalb des unteren und oberen Wertes. Es ist eine Wahrscheinlichkeit, wie beim Würfeln, bei dem nicht jeder 6. Wurf eine 5 zum Beispiel ist, können im Einzelfall einer Messung mehr oder weniger Werte als 5\% außerhalb der beiden Grenzen liegen [Altman 1991, Sachs 1999].

\subsubsection{Sensitivität, Spezifität, positiver und negativer prädiktiver Wert}

Zur Beantwortung der zweiten Fragestellung, ob die Sonographie die Ausdehnung der Entzündung bei Patienten mit einem Morbus Crohn korrekt einschätzen kann, sind die Anwendung der Begriffe Sensitivität und Spezifität geeignet. Ihre Anwendung setzt voraus, dass nur zwei Zustände betrachtet werden: das betreffende Darmsegment ist entzündet oder es ist nicht entzündet. Der Score 1 entspricht dem Ausschluss einer Entzündung und die Scores 2 bis 4 werden als positiver Entzündungsnachweis zusammengefasst.

Damit ergeben sich als richtig positive Resultate die Darmsegmente, die den sonographischen Score 2, 3 oder 4 aufweisen und endoskopisch nicht den Score 1 erhalten. Die richtig negativen Resultate sind die Darmsegmente, die sonographisch und endoskopisch den Score 1 zeigen. Falsch positiv sind die Darmsegmente, die sonographisch mit dem Score 2, 3 oder 4 bewertet werden, jedoch endoskopisch den Score 1 zeigen. Falsch negativ sind die Resultate, die sonographisch den Score 1 bekommen, jedoch endoskopisch den Score 2, 3, oder 4 aufweisen.

Die Sensitivität errechnet sich aus dem Quotienten der richtig positiven Resultate zur Summe der richtig positiven und falsch negativen Ergebnisse. Die Spezifität entspricht dem Quotienten aus den richtig negativen zur Summe aus den richtig negativen und falsch positiven Ergebnissen. 
Der positive prädiktive Wert ist der Quotient der richtig positiven zur Summe aus richtig und falsch positiven Resultaten. In Bezug auf die vorliegende Studie ist er ein Maß für die Wahrscheinlichkeit, dass ein Darmsegment durch den Morbus Crohn tatsächlich entzündlich verändert ist, falls die Sonographie dies voraussagt.

Der negative prädiktive Wert errechnet sich als der Quotient aus den richtig negativen Ergebnissen zur Summe aus den richtig und falsch negativen Ergebnissen. In Bezug auf die vorliegende Studie ist er ein Maß für die Wahrscheinlichkeit, dass ein Darmsegment tatsächlich keine entzündliche Veränderung aufweist, falls die Sonographie es als nicht entzündet beschreibt [Altman 1991, Sachs 1999]. 


\section{Ergebnisse}

\subsection{Power-Dopplersonographie und Ileokoloskopie in der Aktivitätsbewertung}

Insgesamt wurden 180 Darmsegmente bei 31 Patienten mit einem Morbus Crohn sonographisch und endoskopisch untersucht. Auf Grund von Darmresektionen bei insgesamt 4 Patienten entfielen in der Beurteilung 6 Segmente, viermalig das Coecum, einmalig das Colon ascendens sowie einmalig das terminale lleum. Bei 3 von diesen 4 Patienten war es möglich, das neoterminale lleum sonographisch und endoskopisch zu evaluieren.

Die gewichteten Kappa-Koeffizienten waren: 0.82 (95\%-Konfidenzintervall: 0.65 bis 0.98) für das Sigma; 0.90 (95\%-Konfidenzintervall: 0.83 bis 0.98) für das Colon descendens; 0.81 (95\%-Konfidenzintervall: 0.62 bis 0.99) für das Colon transversum; 0.79 (95\%-Konfidenzintervall: 0.62 bis 0.96) für das Colon ascendens; 0.81 (95\%Konfidenzintervall 0.67 bis 0.95) für das Coecum und 0.86 (95\%-Konfidenzintervall: 0.77 bis 0.94 ) für das terminale Ileum.

Die beste Übereinstimmung zwischen der Beurteilung der Aktivität durch die PowerDopplersonographie im Vergleich mit der lleokoloskopie fand sich im Colon descendens. 
Eine Zusammenfassung der Ergebnisse zeigt die Fehler! Keine gültige Verknüpfung..

Tabelle 4: Ergebnisse der Power-Dopplersonographie im Vergleich zur lleokoloskopie

\begin{tabular}{|l|c|c|c|c|c|c|}
\hline & Sigma & $\begin{array}{c}\text { Colon } \\
\text { descendens }\end{array}$ & $\begin{array}{c}\text { Colon } \\
\text { transversum }\end{array}$ & $\begin{array}{c}\text { Colon } \\
\text { ascendens }\end{array}$ & Coecum & $\begin{array}{c}\text { Terminales } \\
\text { lleum }\end{array}$ \\
\hline $\begin{array}{l}\text { Identischer } \\
\text { Score }\end{array}$ & $78 \%$ & $81 \%$ & $81 \%$ & $70 \%$ & $65 \%$ & $60 \%$ \\
\hline $\begin{array}{l}\text { Score- } \\
\text { Differenz: } 1\end{array}$ & $19 \%$ & $19 \%$ & $16 \%$ & $27 \%$ & $35 \%$ & $40 \%$ \\
\hline $\begin{array}{l}\text { Score- } \\
\text { Differenz: } 2\end{array}$ & $3 \%$ & $0 \%$ & $3 \%$ & $3 \%$ & $0 \%$ & $0 \%$ \\
\hline $\begin{array}{l}\text { Score- } \\
\text { Differenz: } 3\end{array}$ & $0 \%$ & $0 \%$ & $0 \%$ & $0 \%$ & $0 \%$ & $0 \%$ \\
\hline $\begin{array}{l}\text { Kappa } \\
\text { gewichtet }\end{array}$ & $\mathbf{0 , 8 2}$ & $\mathbf{0 , 9 0}$ & $\mathbf{0 , 8 1}$ & $\mathbf{0 , 7 9}$ & $\mathbf{0 , 8 1}$ & $\mathbf{0 , 8 6}$ \\
\hline $\begin{array}{l}\text { 95\%- } \\
\text { Konfidenz- } \\
\text { intervall }\end{array}$ & $0,65-0,98$ & $0,83-0,98$ & $0,62-0,99$ & $0,62-0,96$ & $0,67-0,95$ & $0,77-0,94$ \\
\hline
\end{tabular}

Es gab keinen Fall bei den 180 miteinander verglichenen Darmsegmenten, bei dem die größtmögliche Differenz von 3 zwischen den beiden diagnostischen Methoden auftrat. In 3 Fällen (3/180, also 1,6 \%) betrug die Score-Differenz 2, einmalig im Sigma, einmalig im Colon transversum sowie einmalig im Colon ascendens. In diesen 3 Fällen konnte die Sonographie keine entzündlichen Veränderungen detektieren, sie evaluierte diese Darmsegmente als Score 1, die endoskopisch mit dem Score 3 beurteilt wurden. Die umgekehrte Situation, eine sonographische Überschätzung der entzündlichen Aktivität um eine Score-Differenz von 2, fand nicht statt. Eine Score-Differenz von 1 trat in 46 Fällen (46/180, also 25,6 \%) auf. Dabei überschätzte die Sonographie die entzündliche Aktivität in 24 Fällen (24/180, also 13,3 \%) und unterschätzte die Aktivität in 22 Fällen (22/180, also 12,2 \%).

Ein identischer Score von Power-Dopplersonographie und Ileokoloskopie fand sich bei 131 Fällen (131/180, also 72,8 \%). 
Die Verteilung der Scores stellt die Tabelle 5 dar.

Tabelle 5: Score-Resultate von Power-Dopplersonographie und lleokoloskopie

\begin{tabular}{|l|c|c|c|c|c|}
\hline & \multicolumn{5}{|c|}{ leokoloskopie } \\
\hline Sonographie & Score : 1 & Score : 2 & Score : 3 & Score: 4 & Gesamt \\
\hline Score : 1 & 92 & 14 & 3 & 0 & 109 \\
\hline Score : 2 & 6 & 12 & 6 & 0 & 24 \\
\hline Score : 3 & 0 & 8 & 13 & 2 & 23 \\
\hline Score : 4 & 0 & 0 & 10 & 14 & 24 \\
\hline Gesamt & 98 & 34 & 32 & 16 & 180 \\
\hline
\end{tabular}

Drei sonographische Bilder als Beispiele ausgewählt aus den 131 identischen Evaluierungen zeigen die Abbildungen 3 bis Fehler! Keine gültige Verknüpfung..

In der Abbildung 3 sind vereinzelte Gefäße zu sehen, die sich in der echoreichen Submucosa des Colon descendens befinden. Dieses Bild ist ein Beispiel für den Score 2.

Gefäße, die sich intramural in der verdickten Wand des terminalen lleums aufzweigen, stellt die Abbildung 4 dar. Mit der Power-Dopplersonographie wurde dieses Darmsegment als Score 3 evaluiert.

Ein Beispiel für den sonographischen Score 4 ist mit der Abbildung 5 demonstriert. Hier zeigen sich lumenstarke zuführende Gefäße, die sich nach dem Eintritt in die verdickte Darmwand des Colon ascendens bei Darstellung eines kräftigen Dopplersignals in der Submucosa verzweigen. 


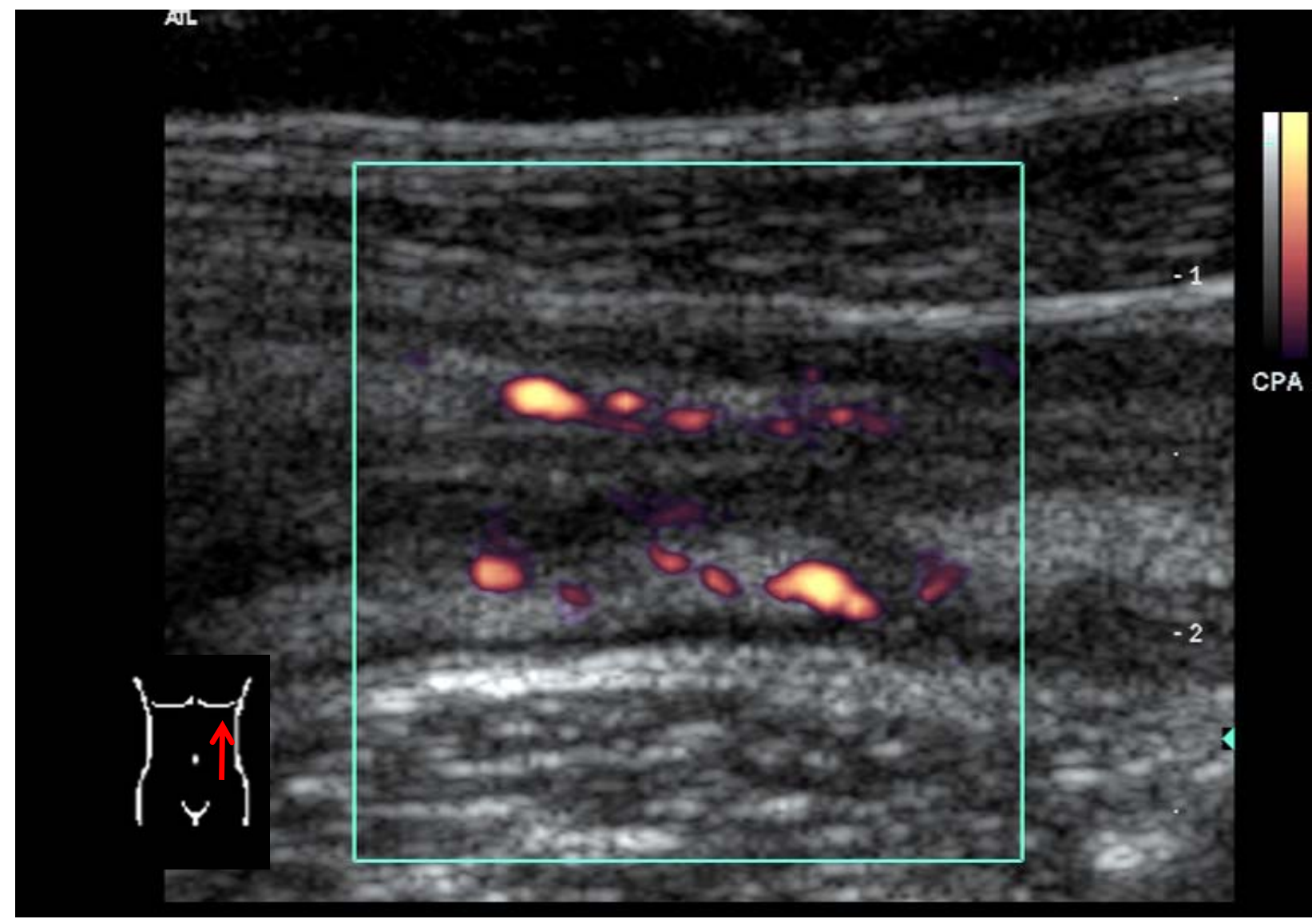

Abbildung 3: Vereinzelte Gefäße in der echogenen Submucosa des Colon descendens: Score 2 


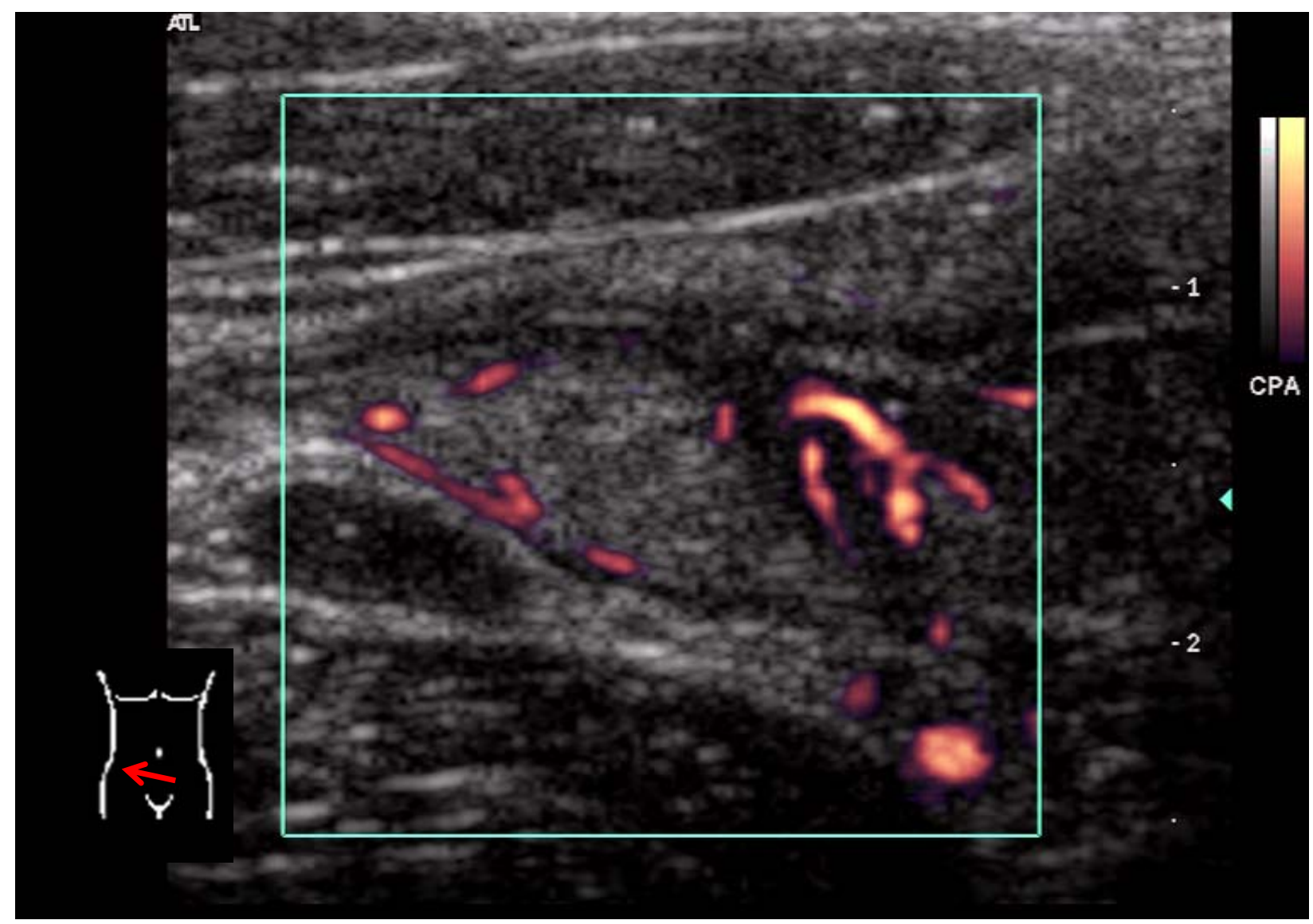

Abbildung 4: Gefäße, die sich in der verdickten Wand des terminalen lleums aufzweigen: Score 3 


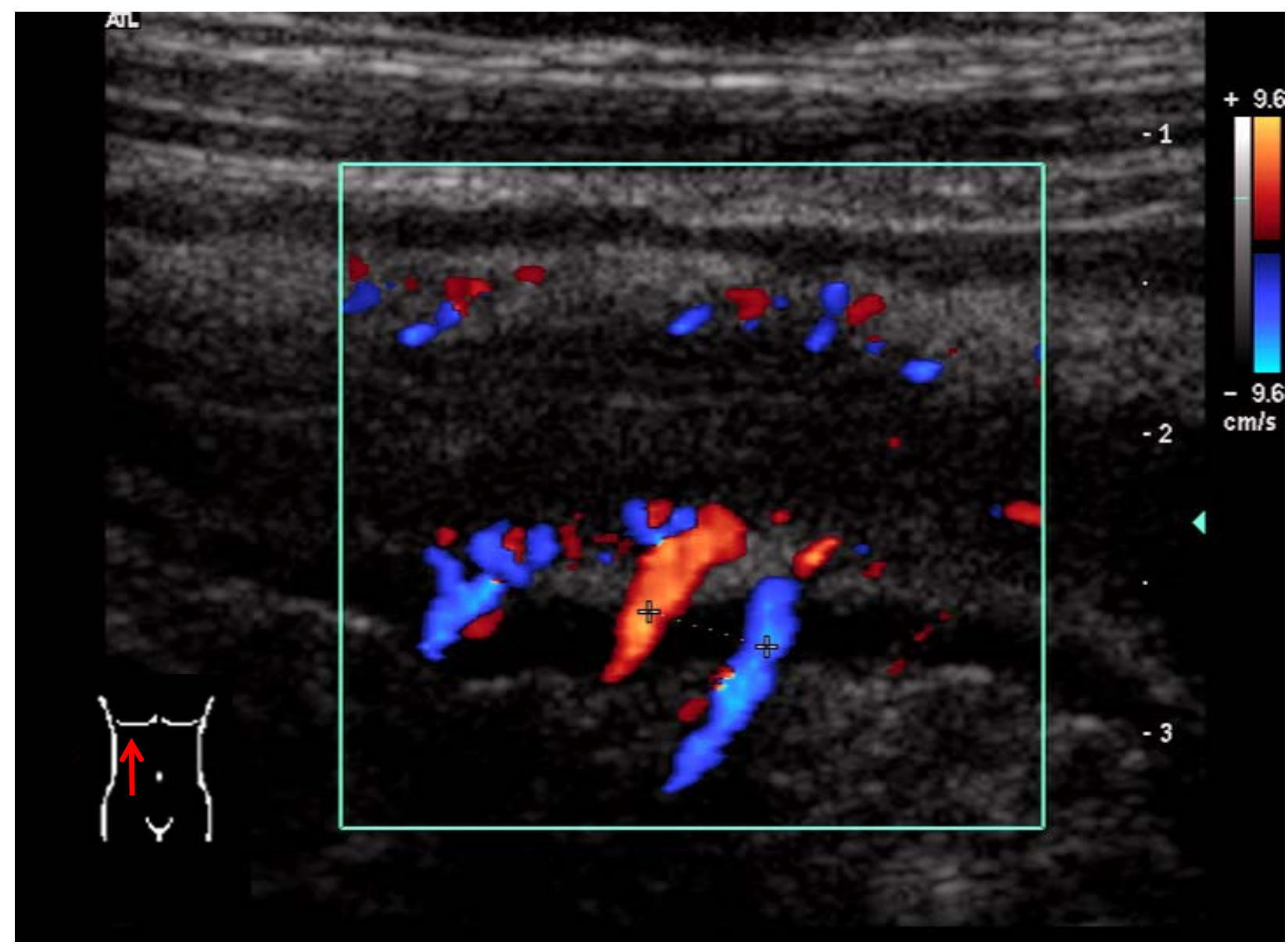

Abbildung 5: Hypervaskularisation mit Nachweis zuführender Gefäße, die die Wand des Colon ascendens penetrieren: Score 4. Das Flusssignal ist kräftig, der Farbdoppler detektiert die Richtung.

\subsection{Sensitivität und Spezifität der Power-Dopplersonographie in der Beurteilung der Ausdehnung der Entzündung bei Patienten mit einem Morbus Crohn}

Wird der Score 1 als Ausschluss einer Entzündung betrachtet und die Scores 2 bis 4 als positiver Entzündungsnachweis subsumiert, kann die Sensitivität und Spezifität der Power-Dopplersonographie zur Beurteilung der Ausdehnung des Morbus Crohns errechnet werden.

Von den 180 evaluierten Darmsegmenten waren 92 richtig negativ (92/180, also $51,1 \%)$. Bei diesen 92 Fällen lag mit beiden Methoden die Einschätzung eines Scores 1 vor. Mit der Power-Dopplersonographie als richtig positiv detektiert wurden 65 Fälle (65/180, also 36,1\%). In diesen Fällen wurde das betreffende Darmsegment sonographisch als entzündet eingestuft, es erhielt den Score 2, 3 oder 4 und wurde endo- 
skopisch nicht mit einem Score 1 bewertet, also die lleokoloskopie fand tatsächlich entzündliche Veränderungen.

Falsch positiv waren 6 Fälle (6/180, also 3,3\%), es lag keine Entzündung vor, die Ileokoloskopie bewertete diese Darmsegmente mit einem Score 1. In allen diesen 6 Fällen stufte die Sonographie die betreffenden Darmsegmente mit einem Score 2 ein, ein Fehler mit einer höheren Score-Differenz trat bei den falsch positiven Befunden nicht auf.

Falsch negativ waren 17 Fälle (17/180, also 9,4\%), sie wurden sonographisch mit einem Score 1 bewertet, obwohl endoskopisch entzündliche Veränderungen nachweisbar waren. 14 dieser 17 Fälle (14/180, also 7,8\%) erhielten den endoskopischen Score 2 und drei Darmsegmente dieser falsch negativen Fälle (3/180, also 1,6\%) wurden mit dem endoskopischen Score 3 bewertet. Eine Score-Differenz von 3 trat nicht auf.

Zusammenfassend verteilen sich die 180 evaluierten Darmsegmente auf 92 richtig negative (51.1\%), 65 richtig positive (36.1\%), 6 falsch positive (3,3\%) sowie 17 falsch negative Resultate $(9,4 \%)$ in der Bewertung durch die Power-Dopplersonographie im Vergleich zur lleokoloskopie.

So ergeben sich für die Power-Dopplersonographie eine Sensitivität von $79,3 \%$ und eine Spezifität von 93,9\% in der Beurteilung, ob ein Darmsegment in den Entzündungsprozess eingeschlossen ist oder nicht. Damit beträgt der positive prädiktive Wert 91,5\% und der negative prädiktive Wert beträgt $84,4 \%$.

\subsection{Die Ergebnisse des Vergleichs mit dem klinischen Aktivitätsindex CDAI}

Die Tabelle 6 spiegelt die Ergebnisse des Vergleichs der sonographischen ScoreSumme eines Patienten mit seinem klinischen Aktivitätsindex CDAl wider. Aus der Tabelle ist ersichtlich, dass nur 16 der 31 Patienten $(51,6 \%)$ in konkordante Gruppen eingeteilt wurden, wobei 3 der Patienten sowohl sonographisch als auch nach der Berechnung des CDAl in einer Remission sich befanden. Bei 13 Patienten liegt sowohl nach der Berechnung der sonographischen Score-Summe als auch nach der CDAIBewertung eine aktive Erkrankung vor. Bei 15 der 31 Patienten $(48,4, \%)$ ist die Einteilung diskordant. Vier Patienten haben laut CDAI eine Remission, sind jedoch gemäß 
ihrer Score-Summe in einer aktiven Erkrankung. Bei 11 der 31 Patienten liegt der umgekehrte Fall vor, ihr sonographischer Score weist keine aktive Erkrankung nach, laut ihrem CDAl befinden sie sich jedoch in dieser.

Zusammenfassend kann gesagt werden, die sonographische Score-Summe und die Bewertung der klinischen Krankheitsaktivität durch den CDAl korrelieren nicht ausreichend miteinander.

Tabelle 6: Vergleich der sonographischen Score-Summe mit dem CDAI

\begin{tabular}{|c|c|c|c|}
\hline & \multicolumn{3}{|c|}{ CDAI } \\
\hline Score-Summe & $\leq 150$ & $>150$ & Gesamt \\
\hline$\leq 9$ & $\mathbf{3}$ & 11 & 14 \\
\hline$>9$ & 4 & $\mathbf{1 3}$ & 17 \\
\hline Gesamt & 7 & 24 & $\mathbf{3 1}$ \\
\hline
\end{tabular}




\section{Diskussion}

Patienten mit einem Morbus Crohn leiden an einer Erkrankung, deren charakteristische Merkmale, der unbestimmte Verlauf, die unbestimmte Ausdehnung und die unbestimmte Aktivität sind.

Entscheidungen zur internistischen oder chirurgischen Therapie der Erkrankung setzen eine Diagnostik voraus, die die Ausdehnung und die Aktivität einzelner Darmsegmente bestimmen kann. Im Bereich des Colons und des terminalen lleums ist dies die lleokoloskopie, die jedoch als eine invasive Methode mit Nebenwirkungen verbunden ist, wodurch sie nicht beliebig oft einsetzbar ist.

Zur Beantwortung der ersten Aufgabenstellung der vorliegenden Arbeit soll geklärt werden, ob die Einschätzung der entzündlichen Aktivität einzelner Darmsegmente durch die Sonographie unter Einbeziehung der neuen Dopplermethoden im Vergleich mit der lleokoloskopie zu ähnlichen oder zu divergenten Ergebnissen führt.

Eine nicht-invasive Untersuchungsmethode, die keine ionisierende Strahlung anwendet, ist zur Verlaufskontrolle in der Beurteilung der Entzündungsaktivität des Morbus Crohns wünschenswert. Der Wunsch, die Sonographie hierfür einzusetzen, wurde bereits früh publiziert [Holt et al. 1979, Kimmey et al. 1989, Meckler et al.1991, Hata et al. 1992].

Der Morbus Crohn ist charakterisiert durch eine segmental auftretende Entzündung des Gastrointestinaltraktes, die rezdivierend in Schüben auftritt. Zur Einschätzung der lokalen Entzündungsaktivität sind endoskopische Untersuchungen der Standard [Miao et al. 2002, Schölmerich 2003].

In einer pädiatrischen Studie zeigte sich eine gute Korrelation zwischen der Darmwandverdickung, die der Morbus Crohn verursachte, und seiner entzündlichen Aktivität [Haber et al. 2000]. Auch spätere Studien bei Kindern, die an einem Morbus Crohn erkrankten, konnten die guten Ergebnisse der B-Mode-Sonographie bestätigen [Caiulo et al. 2003, Alison et al. 2007, Strong et al. 2007]. Diese gute Korrelation zwischen der Dicke der Darmwand und der Entzündungsaktivität, die bei Kindern mit einem Morbus Crohn in diesen Arbeiten gezeigt wurden, lässt sich auf Erwachsene nicht übertragen. Zwischen der Darmwandverdickung und der Entzündungsaktivität besteht bei erwachsenen Patienten mit einem Morbus Crohn nur eine schwache Korrelation [Maconi 
et al. 1996]. In dieser Studie fanden sich Patienten in einer inaktiven Krankheitsphase mit zum Teil ausgedehnten Verdickungen der Darmwand. Die Fähigkeit der reinen BBild-Sonographie zur Unterscheidung von aktiven und inaktiven Krankheitsphasen wird durch sehr ähnliche Befunde von inflammatorischen und fibrosierenden Verdickungen der Darmwand limitiert.

Wenn der Unterschied zwischen der Korrelation von Wanddicke und Entzündungsaktivität, die laut den publizierten Studien bei Kindern gut und bei erwachsenen Patienten schwach ist, diskutiert werden soll, dann kann ein Zusammenhang mit dem Patientenalter sowie der Krankheitsdauer vermutet werden. Fibrosierungen sind bei Kindern deutlich seltener als bei Erwachsenen mit einem Morbus Crohn.

Messungen des Blutflusses in der Arteria mesenterica superior und inferior durch Dopplermessungen sind als eine weitere Möglichkeit beschrieben worden, die Krankheitsaktivität von Patienten mit Morbus Crohn zu bestimmen [Van Oostayen et al. 1994, 1997, 1998]. Diese Untersuchungstechnik wird durch die Winkelabhängigkeit des Dopplershifts sowie ausgeprägter inter- und intraindividueller Streubreite der Messungen limitiert [Zoli et al. 1996]. In späteren Studien zeigten Dopplermessungen der Mesenterialarterien keinen statistisch signifikanten Unterschied zwischen Patienten mit einem aktiven und einem inaktiven Morbus Crohn [Miao et al. 2002]. Die Bedeutung der Dopplermessungen der Arteria mesenterica superior und inferior für die Beurteilung der Krankheitsaktivität wird kontrovers beurteilt [Ludwig et al. 1999, 2004, Yekeler et al. 2005], eine Bestimmung der entzündlichen Aktivität einzelner Darmsegmente kann jedoch in keinem Fall von Messungen an den großen Viszeralarterien erwartet werden.

Die Angiographie war bis zur Entwicklung neuer Ultraschalltechniken die einzige diagnostische Methode mit der eine Darstellung der intramuralen Gefäße des Darmes möglich war. In diesen Arbeiten konnte gezeigt werden, dass aktive Krankheitsphasen mit einer Hypervaskularisation der Darmwandgefäße assoziiert sind [Bousen et al. 1966, Lunderquist 1967]. Die Bedeutung dieser angiographischen Beobachtung der 60iger Jahre des letzten Jahrhunderts konnte sonographisch erst genutzt werden, als es technisch möglich wurde, den langsamen Blutfluss in den kleinen Darmwandgefäßen darzustellen. 
So wurde eine Kombination von B-Mode und der Power-Dopplersonographie zur Beurteilung der Entzündungsaktivität vorgeschlagen [Heyne et al. 2002]. Der Hintergrund für diesen Vorschlag war der Erfolg der neuen Ultraschallmethoden in der Detektion von langsamen Blutflüssen in kleinen Gefäßen bei anderen Körperregionen [Wermke et al. 1998, Rickes et al. 2000, 2002].

Mehrere Studien [Esteban et al. 2003, Maconi et al. 2003, 2006, 2007, Parente et al. 2004, 2005, Castiglione et al. 2004, Kratzer et al. 2004, Kunihiro et al. 2004, Fraquelli et al. 2005, Mackalski et al. 2006] untersuchten bereits den Wert von Ultraschalltechniken für die Beurteilung der Entzündungsaktivität.

Zwei Besonderheiten besitzt die vorliegende Studie gegenüber den genannten. Zum einen werden einzelne Darmsegmente paarweise sonographisch und endoskopisch untersucht und nicht nur der einzelne Patient der gesamten Entzündungsaktivität seines Darmes zugeordnet. Damit fließt auch die korrekte Zuordnung des Ortes der Entzündung in die Bewertung der sonographischen Qualität ein. Ordnet der sonographierende Untersucher zwei Darmsegmente genau umgekehrt zur Endoskopie ein, senken beide Evaluationen die statistischen Parameter zu Ungunsten des Ultraschalls. Das Studiendesign der vorliegenden Arbeit imitiert damit stärker den klinischen Alltag. Ein Patient sucht ärztliche Hilfe selten ohne jegliche Beschwerden bei völliger Gesundheit auf. Würde der Ultraschall bei allen diesen Patienten einfach eine moderate Entzündungsaktivität voraussagen, wären auch die globalen Parameter bezüglich des Patienten statistisch moderat. Anders ist es in der vorliegenden Studie, da hier 6 Darmsegmente jedes Patienten voneinander unabhängig in der statistischen Auswertung berücksichtigt werden.

Die zweite Besonderheit liegt in der Graduierung der Entzündungsaktivität mit vier Kategorien. Damit werden die 180 Darmsegmente nicht nur einem entzündeten oder nicht entzündeten Zustand zugeordnet. In diesem Fall wären 180 × 2 Bewertungen möglich, also 360. Bei vier Kategorien sind 180 × 4 Bewertungen möglich, also insgesamt 720. Auch dieses Studiendesign reflektiert stärker den klinischen Alltag, sind oft für medizinische Entscheidungen doch graduelle Unterschiede ausschlaggebend. Würde bei neuen Medikamenten für eine Dosisänderung so lange gewartet werden, bis eine Entzündung vollständig abgeklungen bzw. zur massiven Exazerbation führt, wäre das Monitoring durch den Ultraschall oft nicht besser als durch globale Entzündungs- 
parameter, die in einer Blutprobe erkennbar sind [Arienti et al. 2000, Hirche et al. 2002, Pascu et al. 2004, Hagiu et al. 2007].

Die vorliegende Arbeit zeigte, dass die Einschätzung der entzündlichen Aktivität einzelner Darmsegmente durch die Sonographie unter Einbeziehung der neuen Dopplermethoden im Vergleich mit der lleokoloskopie zu konkordanten Ergebnissen führt (siehe Tabelle 4). Dabei zeigten die Ergebnisse im Colon descendens die größte Übereinstimmung. Soll der Grund hierfür diskutiert werden, kann eine gute Zugänglichkeit dieser Region angeführt werden, woraus ein Vorteil für den sensitiven Powerdoppler resultiert. Trotz potentiell 720 möglicher Zuordnungen (siehe oben) konnte der Ultraschall in 131 von 180 Fällen (131/180, also 72,8\%) die Entzündungsaktivität im Vergleich zur lleokoloskopie korrekt zuordnen. Eine grundsätzliche Tendenz zur Über- bzw. Unterbewertung der Aktivität durch den Ultraschall dokumentiert die vorliegende Studie nicht, da die Score-Differenz von 1 in 46 Fällen auftretend, sich auf 24 Überbewertungen (24/46, also 52,2\%) und 22 Unterbewertungen (22/46, also 47,8\%) aufteilt. Die schlechtesten Bewertungen durch die Sonographie mit einer Score-Differenz von 2 lassen sich nicht einer definierten Region zuordnen, da sie jeweils einmalig an unterschiedlichen Darmsegmenten auftraten (Sigma, Colon transversum, Colon descendens).

Grundsätzlich zeigen die Ergebnisse der Studie mit den zuvor veröffentlichten Daten der bereits zitierten Angiographie- und Ultraschallstudien vergleichbare Resultate, entzündete Darmsegmente sind hypervaskularisiert, keine oder nur wenige Gefäße fanden sich in den nicht entzündlich veränderten Darmsegmenten (siehe Tabelle 5).

Diese Studie bewies auch, dass die Power-Dopplersonographie Gefäße unter 1mm Durchmesser detektieren kann und auch irregulär sowie geschlängelt verlaufende Gefäße innerhalb der Darmwand darzustellen vermag (siehe Abbildungen 3 bis 5). Zukünftig könnte die direkte Darstellung der intramuralen Darmgefäße der größte Vorteil des Ultraschalls gegenüber den anderen bildgebenden Verfahren darstellen.

Auch in der Beantwortung der zweiten Fragestellung zeigte sich, dass die Sonographie die Ausdehnung der Entzündung bei Patienten mit einem Morbus Crohn korrekt einschätzen kann, wobei ihre Spezifität mit 93,9\% höher als ihre Sensitivität mit 79,3\% ist. 
In den bereits zitierten Studien wird die Sensitivität des Ultraschalls mit $56 \%$ bis maximal 96\% angegeben, seine Spezifität von 83\% bis maximal 100\%.

Insbesondere geringgradige Entzündungen der Mucosa, die sich im endoskopischen Bild mit einzelnen Aphthen präsentieren, entziehen sich häufiger als ausgeprägte entzündliche Veränderungen dem sonographischen Nachweis (siehe Tabelle 5). Diesen Zusammenhang spiegeln auch die prädiktiven Werte wider. Der positive prädiktive Wert ist mit 91,5\% höher als der negative mit 84,4\%, der Nachweis einer Entzündung durch den Ultraschall ist sicherer als der sonographische Ausschluss.

In den bereits zitierten Studien wird der positiv prädiktive Wert des Ultraschalls mit 77\% bis maximal 100\% angegeben, wobei deutlich weniger Autoren die prädiktiven Werte publizierten als die Sensitivität und Spezifität. Der negative prädiktive Wert des Ultraschalls zeigt in diesen Arbeiten eine Schwankung von minimal 57\% bis maximal 96\%.

Damit erbrachte diese Studie zu den vorliegenden Publikationen grundsätzlich vergleichbare Ergebnisse. Wie aus der Tabelle 5 jedoch ersichtlich, konnte die Aufteilung in Kategorien in der vorliegenden Arbeit zeigen, dass eine Minderung der Sensitivität sowie des negativ prädiktiven Wertes überwiegend durch das falsche Einordnen des endoskopischen Scores 2 als nicht entzündet durch die Sonographie verursacht wird.

Um die Sensitivität und die Spezifität der Darmsonographie der vorliegenden sowie der publizierten Studien im klinischen Alltag erreichen zu können, spielt neben einer guten Geräteausstattung mit hochsensitiven Farb- und Powerdopplern und hochfrequenten Linearscannern auch die sonographische Erfahrung des Untersuchers eine herausragende Rolle. Im Gegensatz zu CT oder MRT sind nachträgliche Befundungen von einem Untersucher mit größerer Erfahrung nicht durchführbar. Der Erstuntersucher kann und muss mit seiner Hand die jeweilige Schnittebene des Bildes willkürlich aus unendlich vielen Winkeln der drei Grundebenen des Raumes auswählen. Seine möglicherweise fehlerhafte Bildauswahl mit fehlerhafter Interpretation ist, sobald der Patient die Untersuchungsliege verlassen hat, von einem erfahreneren Untersucher nicht mehr in vollem Umfang korrigierbar. Anderseits ermöglicht diese Freiheit in der Auswahl der Schnittebene verbunden mit der sehr guten Ortsauflösung des Ultraschalls eine hohe Anpassung des Schnittbildes an den jeweiligen Patienten mit seinem individuellen 
Krankheitsbild. Insbesondere bei Patienten mit einem Morbus Crohn gestattet diese individuelle Anpassungsfähigkeit des Ultraschalls eine gute Befundgenauigkeit, da der Darm interindividuell eine sehr unterschiedliche Lage im menschlichen Körper hat und die Erkrankung diskontinuierlich zu Entzündungen führt.

Für die Beurteilung von Komplikationen wie Fistelbildung und Abszedierungen spielen für Patienten mit einem Morbus Crohn auch andere bildgebende Verfahren eine wichtige Rolle. So besitzt die Computertomographie die Vorteile der nachträglichen Befundung durch einen erfahrenen Untersucher, ihre gute Anwendbarkeit auch bei Meteorismus und Adipositas sowie die Untersuchbarkeit des Patienten vom Thorax bis in das Becken mit nur einem bildgebenden Verfahren. Ihr bedeutendster Nachteil ist die Strahlenbelastung für den Patienten, wodurch die Häufigkeit von Verlaufskontrollen insbesondere auch durch das eher jüngere Alter der Patienten mit Morbus Crohn deutlich limitiert ist. Die Magnetresonanztomographie ist im Gegensatz zur Sonographie oder auch dem CT in der Akutdiagnostik häufig nicht verfügbar, ihre besondere Bedeutung für Patienten mit Morbus Crohn liegt in der Beurteilung von Komplikationen im Bereich des kleinen Beckens. Von diesen drei bildgebenden Verfahren besitzt die Sonographie die höchste Ortsauflösung und als einzige Bildgebung die Darstellbarkeit von einzelnen Gefäßen innerhalb der Darmwand mit arteriellen bzw. venösen Spektren. Im klinischen Alltag sind diese Verfahren mit ihren verschiedenen Optionen komplementär einsetzbar [Parente et al. 2002, Maconi et al 2003, Neye et al. 2004, Pascu et al. 2004].

Der Vergleich der sonographischen Score-Summe mit dem klinischen Aktivitätsindex CDAl ergab in der vorliegenden Studie, dass diese nicht ausreichend miteinander korrelieren. Dieses Ergebnis steht im Einklang mit bisherigen Veröffentlichungen [Heyne et al. 2002, Hirche et al. 2002, Maconi et al 2003]. Die Schwäche des CDAl zur Abschätzung der Krankheitsaktivität ist auch in der S3-Leitlinie „Diagnostik und Therapie des Morbus Crohn“ der Deutschen Gesellschaft für Verdauungs- und Stoffwechselkrankheiten dokumentiert, die als wichtigste Untersuchungsmethode zur Bestimmung des Ausmaß der Entzündung und des Befallsmuster die Koloskopie nennt [Hoffmann et al. 2008]. Sollen die Hintergründe für die schwache Korrelation diskutiert werden, so sind auch die subjektiven Angaben des Patienten zu betrachten, die die Höhe des CDAI definieren. Der Grad der Bauchschmerzen und die Beeinträchtigung des Allgemeinbefindens eines Patienten werden nicht nur von der aktuellen Entzündung 
der Darmwand determiniert. So führen abgelaufene Entzündungen zu Verwachsungen und Knickbildungen des Darmes, die sehr schmerzhafte Stuhlpassagen ohne Vorliegen einer erneuten Entzündung verursachen können. Im Extremfall wird das Lumen so durch eine Bride komprimiert, dass ein lleus resultiert, der nur chirurgisch behandelbar ist. Auf der anderen Seite sind Patienten mit einem Morbus Crohn auch als chronische Schmerzpatienten zu betrachten, bei denen trotz Wegfall der auslösenden Noxe der Schmerz persistieren kann. Die Besonderheit des Morbus Crohn liegt desweiteren in seinen extraintestinalen Manifestationen, so kann z.B. eine Sacroileitis als ein akuter Schub fehlgedeutet werden [Hata et al. 1994, Hirche et al. 2002]. Auch eine erhöhte Anzahl der weichen Stühle der letzten Woche, die in den CDAI einfließt, muss nicht nur durch eine Entzündung der Darmwand selbst verursacht sein. Überlagerungen durch funktionelle Störungen, assoziierte Nahrungsmittelunverträglichkeiten oder Sekundärinfektionen treten häufig bei Patienten mit einem Morbus Crohn auf [Hoffmann et al. 2008]. So kann gesagt werden, dass die objektiven Methoden zur Einschätzung der Entzündungsaktivität der Darmwand nicht durch klinische Indizes wie den CDAI ersetzt werden können.

Gegenwärtig bleibt noch offen, ob die weitere Entwicklung des Ultraschalls zu noch sensitiveren Farb- bzw. Powerdopplern als heute eine weitere Verbesserung in der Beurteilung der Entzündungsaktivität bei Patienten mit einem Morbus Crohn bewirken wird.

Schon jetzt zeigen die Ergebnisse dieser Studie eine hohe Konkordanz der nichtinvasiven Kombination von B-Mode mit der Power-Dopplersonographie im Vergleich mit der lleokoloskopie, dem gegenwärtig diagnostischen Standard.

Aus den Ergebnissen der vorliegenden Arbeit ergibt sich, dass der Ultraschall unter Anwendung der Power-Dopplersonographie zur Bestimmung der Krankheitsaktivität als Verlaufskontrolle bei Patienten mit Morbus Crohn geeignet ist.

Beim transabdominellen Ultraschall sind keine ernsthaften Nebenwirkungen bekannt. Weitere Vorteile sind seine vergleichsweise niedrigen Kosten und seine potenziell gute Verfügbarkeit. Die Sonographie kann jedoch die histologische Sicherung, die während einer Endoskopie durchgeführt werden kann, nicht ersetzen. Auch besitzt die 
Ileokoloskopie zum Ausschluss eines Kolonkarzinoms eine weiterhin essenzielle Bedeutung.

So kann abschließend die Sonographie als eine zur Endoskopie komplementäre Untersuchungsmethode in der Hand des erfahrenen Gastroenterologen betrachtet werden.

\section{Zusammenfassung}

In der Literatur finden sich bisher nur wenige prospektive Daten zum klinischen Wert der Power-Dopplersonographie bei Morbus Crohn. Der Goldstandard zur Bewertung der Aktivität ist die Ileokoloskopie. Ziel dieser prospektiven Studie war die Evaluierung der Power-Dopplersonographie mit Beantwortung folgender Fragen:

1. Führt die Einschätzung der entzündlichen Aktivität einzelner Darmsegmente durch die Sonographie unter Einbeziehung der neuen Dopplermethoden im Vergleich mit der lleokoloskopie zu konkordanten oder zu divergenten Ergebnissen?

2. Kann die Sonographie die Ausdehnung der Entzündung bei Patienten mit einem Morbus Crohn korrekt einschätzen?

31 Patienten mit gesichertem Morbus Crohn wurden prospektiv mit dem B-Mode sowie der Power- Dopplersonographie und mit der lleokoloskopie untersucht. Die Sonographie erfolgte binnen 3 Tagen vor der Endoskopie. Sämtliche Untersuchungen führten erfahrene Diagnostiker durch, die zu den klinischen Daten und den Ergebnissen der übrigen Untersuchungsverfahren verblindet waren. Mit Hilfe vor Studienbeginn definierter Kriterien wurde die Aktivität für den Ultraschall und für die Ileokoloskopie für jedes einzelne Darmsegment bestimmt. Die Aktivität wurde nach einem Score von 1 (keine) bis 4 (hohe) eingeteilt. Bei den 31 Patienten wurden insgesamt 180 Darmsegmente sonographisch und endoskopisch untersucht. Die statistische Auswertung erfolgte mit dem gewichteten Kappa-Test und mit der Berechung von Sensitivität, Spezifität sowie den prädiktiven Werten. 
In den Ergebnissen der Studie zeigte sich eine hohe Konkordanz in den Resultaten von Ultraschall und Ileokoloskopie. Die Befunde im Colon descendens stimmten am stärksten überein.

Diese prospektive Studie evaluierte die Power-Dopplersonographie und beantwortete die Fragestellungen wie folgt:

1. Die Einschätzung der entzündlichen Aktivität einzelner Darmsegmente durch die Sonographie unter Einbeziehung der neuen Dopplermethoden im verblindeten Vergleich mit der Ileokoloskopie führt zu konkordanten Ergebnissen.

2. Die Sonographie kann die Ausdehnung der Entzündung bei Patienten mit einem Morbus Crohn korrekt einschätzen, wobei ihre Spezifität höher als ihre Sensitivität ist. Insbesondere geringgradige Entzündungen der Mucosa, die sich im endoskopischen Bild mit einzelnen Aphten präsentieren, entziehen sich häufiger als ausgeprägte entzündliche Veränderungen dem sonographischen Nachweis. Diesen Zusammenhang spiegeln auch die prädiktiven Werte wider. Der positive prädiktive Wert ist höher als der negative, der Nachweis einer Entzündung durch den Ultraschall ist sicherer als der sonographische Ausschluss.

Die Kombination von B-Mode und Power-Dopplersonographie besitzt eine hohe Genauigkeit in der Bewertung der Aktivität bei Morbus Crohn. Der Ultraschall als Kombination von B-Mode und Power-Dopplersonographie in der Hand des erfahrenen Untersuchers kann zu Verlaufsuntersuchungen bei Patienten mit einem Morbus Crohn empfohlen werden. 


\section{Literaturverzeichnis}

Alison M, Kheniche A, Azoulay R, Roche S, Sebag G, Belarbi N. Ultrasonography of Crohn disease in children. Pediatr Radiol 2007;25:1071-82.

Altman DG. Practical Statistics for Medical Research. London, England: Chapman \& Hall/CRC,1991: 406-7.

Ambrosini R, Barchiesi A, Di Mizio V. Inflammatory chronic disease of the colon: how to image. Eur J Radiol 2007; 61:442-8.

Arienti V, Zamboni L, Gionchetti P, Rizzello F, Campieri M. Ultrasonographic findings in Crohn's disease. Gut 2000; 46:293.

Best WR, Becktel JM, Singleton JW, Kern F. Development of a Crohn's disease activity index. National Cooperative Crohn's Disease Study. Gastroenterology 1976; 70:439-44.

Bousen E, Reuter SR. Mesenteric angiography in the evaluation of inflammatory and neoplastic disease of the intestine. Radiology 1966;87:1028-36.

Bude RO, Rubin JM, Adler RS. Power versus conventional color Doppler sonography: comparison in the depiction of normal intrarenal vasculature. Radiology 1994;190:853-6.

Caiulo VA, Latini G, De Felice C. Ultrasound in the diagnosis of Crohn disease in childhood. Eur J Pediatr 2003;162:285-6.

Castiglione F, de Sio I, Cozzolino A, Rispo A et al. Bowel wall thickness at abdominal ultrasound and the one-year-risk of surgery in patients with Crohn's disease. Am J Gastroenterol 2004;99:1977-83.

Chmura Kraemer H, Periyakoil VS, Noda A. Kappa coefficients in medical research. Stat Med $2002 ; 21: 2109-29$.

Esteban JM, Aleixandre A, Hurtado MJ, Maldonado L, Mora FJ, Nogués E. Contrastenhanced power Doppler ultrasound in the diagnosis and follow-up of inflammatory abdominal masses in Crohn's disease. Eur J Gastroenterol Hepatol 2003;15:253-9.

Fraquelli M, Colli A, Casazza G et al. Role of US in detection of Crohn disease: metaanalysis. Radiology 2005;236:95-101.

Graham P, Jackson R. The analysis of ordinal agreement data: beyond weighted kappa. J Clin Epidemiol 1993;46:1055-62. 
Haber HP, Busch A, Ziebach R, Stern M: Bowel wall thickness measured by ultrasound as a marker of Crohn's disease activity in children. Lancet 2000;355:1239-40.

Hagiu C, Badea R. Applicability of abdominal ultrasonography in inflammatory bowel diseases. J Gastrointestin Liver Dis 2007;16:205-9.

Hata J, Haruma K, Suenaga K, Yoshihara M, et al. Ultrasonographic assessment of inflammatory bowel disease. Am J Gastroenterol 1992;87:443-7.

Hata J, Haruma K, Yamanaka H, et al. Ultrasonographic evaluation of the bowel wall in inflammatory bowel disease: comparison of in vivo and in vitro studies. Abdom Imaging 1994;19:395-9.

Heyne R, Rickes S, Bock P, Schreiber S, Wermke W, Lochs H. Non-invasive evaluation of activity in inflammatory bowel disease by power Doppler sonography. Z Gastroenterol 2002;40:171-5.

Hirche TO, Russler J, Schröder O, et al. The value of routinely performed ultrasonography in patients with Crohn disease. Scand J Gastroenterol 2002;37:1178-83.

Hoffmann JC, Preiss JC, Autschbach F, Buhr HJ et al. Clinical practice guideline on diagnosis and treatment of Crohn's disease. Z Gastroenterol 2008; 46: 1094-146.

Holt S, Samuel E. Grey scale ultrasound in Crohn's disease. Gut 1979;20:590-5.

Kimmey MB, Martin RW, Haggitt RC, Wang KY, Franklin DW, Silverstein FE. Histologic correlates of gastrointestinal ultrasound images. Gastroenterology 1989;96:43341.

Kratzer W, Foeller T, Kaechele V, Reinshagen M, Tirpitz CV, Haenle MM. Intestinal wall vascularisation in Crohn's disease. Z Gastroenterol 2004;42:973-8.

Kundel HL, Polansky M. Measurement of observer agreement. Radiology 2003;228:303-8.

Kunihiro K, Hata J, Haruma K, Manabe N, Tanaka S, Chayama K. Sonographic detection of longitudinal ulcers in Crohn disease. Scand J Gastroenterol 2004;39:322-6.

Landis JR, Koch GG. The measurement of observer agreement for categorical data. Biometrics 1977;33:159-74

Ludwig D, Wiener S, Brüning A, et al. Mesenteric blood flow is related to disease activity and risk of relapse in ulcerative colitis: a prospective follow up study. Gut 1999;45:546-52. 
Ludwig D. Doppler sonography in inflammatory bowel disease. Z Gastroenterol 2004;42:1059-65.

Lunderquist A, Knutsson $\mathrm{H}$. Angiography in Crohn's disease of the small bowel and colon. Am J Roentgenol Radium Ther Nucl Med 1967;101:338-44.

Mackalski BA, Bernstein CN. New diagnostic imaging tools for inflammatory bowel disease. Gut 2006;55:733-41.

Maconi G, Parente F, Bollani S, Cesana B, Bianchi Porro G. Abdominal ultrasound in the assessment of extent and activity of Crohn's disease: clinical significance and implication of bowel wall thickening. Am J Gastroenterol 1996;91:1604-9.

Maconi G, Radice E, Greco S, Bezzio C, Bianchi Porro G. Transient small-bowel intussusceptions in adults: significance of ultrasonographic detection. Clin Radiol 2007;62:792-7.

Maconi G, Radice E, Greco S, Bianchi Porro G. Bowel ultrasound in Crohn's disease. Best Pract Res Clin Gastroenterol 2006;20:93-112.

Maconi G, Sampietro GM, Parente F, et al. Contrast radiology, computed tomography and ultrasonography in detecting internal fistulas and intra-abdominal abscesses in Crohn's disease: a prospective comparative study. Am J Gastroenterol 2003;98:1545-55.

Maconi G, Sampietro GM, Russo A, et al. The vascularity of internal fistulae in Crohn's disease: an in vivo power Doppler ultrasonography assessment. Gut 2002;50:496-500.

Martinoli C, Derchi LE, Rizzatto G, Solbiati L: Power Doppler sonography: general principles, clinical applications, and future prospects. Eur Radiol 1998;8:1224-35.

Meckler U, Caspary WF, Clement T, et al. Sonography in Crohn disease- the conclusions of an experts' group. Z Gastroenterol 1991;29:355-9.

Miao YM, Koh DM, Amin Z, et al. Ultrasound and magnetic resonance imaging assessment of active bowel segments in Crohn's disease. Clin Radiol 2002;57:913-8.

Munoz SR, Bangdiwala SI. Interpretation of kappa and B statistic measures of agreement. J Appl Stat 1997;24:105-11.

Newman JS, Adler RS, Bude RO, Rubin JM. Detection of soft-tissue hyperemia: value of power Doppler sonography. Am J Roentgenol 1994;163:385-9. 
Neye H, Voderholzer W, Rickes S, Weber J, Wermke W, Lochs H: Evaluation of Criteria for the Activity of Crohn's Disease by Power Doppler Sonography. Dig Dis 2004;22:67-72.

Parente F, Greco S, Molteni M, Anderloni A, Bianchi Porro G. Imaging inflammatory bowel disease using bowel ultrasound. Eur J Gastroenterol Hepatol 2005;17:28391.

Parente F, Greco S, Molteni M, Anderloni A, Maconi G, Bianchi Porro G. Modern imaging of Crohn's disease using bowel ultrasound. Inflamm Bowel Dis 2004;10:452-61.

Parente F, Maconi G, Bianchi Porro G. Bowel ultrasound in Crohn disease: current role and future applications. Scand J Gastroenterol 2002;37:871-6.

Pascu M, Roznowski AB, Müller HP, Adler A, Wiedenmann B, Dignass AU. Clinical relevance of transabdominal ultrasonography and magnetic resonance imaging in patients with inflammatory bowel disease of the terminal ileum and large bowel. Inflamm Bowel Dis 2004;10:373-82.

Rapaccini GL, Pompili M, Orefice R et al. Contrast-enhanced power doppler of the intestinal wall in the evaluation of patients with Crohn disease. Scand $\mathrm{J}$ Gastroenterol 2004;39:188-94.

Rickes S, Schulze S, Neye H, Ocran KW, Wermke W. Improved diagnosing of small hepatocellular carcinomas by echo-enhanced power Doppler sonography in patients with cirrhosis. Eur J Gastroenterol Hepatol 2003;15:893-900.

Rickes S, Unkrodt K, Neye H, Ocran KW, Wermke W. Differentiation of pancreatic tumours by conventional ultrasound, unenhanced and echo-enhanced power Doppler sonography. Scand J Gastroenterol 2002;37:1313-20.

Rickes S, Unkrodt K, Ocran K, Neye H, Lochs H, Wermke W. Evaluation of doppler ultrasonography criteria for the differential diagnosis of pancreatic tumors. Ultraschall Med 2000;21:253-8.

Rispo A, Bucci L, Pesce $G$ et al. Bowel sonography for the diagnosis and grading of postsurgical recurrence of Crohn's disease. Inflamm Bowel Dis 2006;12:486-90.

Rubin JM, Bude RO, Carson PL, Bree RL, Adler RS. Power Doppler US: a potentially useful alternative to mean frequency-based color Doppler US. Radiology 1994;190:853-6.

Sachs L. Angewandte Statistik: Anwendung statistischer Methoden. 9. Aufl. Berlin, Heidelberg, New York: Springer-Verlag, 1999:472-3. 
Saibeni S, Rondonotti E, lozzelli A et al. Imaging of the small bowel in Crohn's disease: a review of old and new techniques. World J Gastroenterol 2007;13:3279-87.

Schlottmann K, Kratzer W, Schölmerich J. Doppler ultrasound and intravenous contrast agents in gastrointestinal tract disorders: current role and future implications. Eur J Gastroenterol Hepatol 2005;17:263-75.

Scholmerich J. Inflammatory bowel disease. Endoscopy 2003;35:164-70.

Sim J, Wright CC. The kappa statistic in reliability studies: use, interpretation, and sample size requirements. Phys Ther 2005;85:257-68.

Sonnenberg A, Erckenbrecht J, Peter P, Niederau C. Detection of Crohn's disease by ultrasound. Gastroenterology 1982;83:430-4.

Strong SA. Perianal Crohn's disease. Semin Pediatr Surg 2007;16:185-93.

Valette PJ, Rioux M, Pilleul F, Saurin JC, Fouque P, Henry L. Ultrasonography of chronic inflammatory bowel diseases. Eur Radiol 2001;11:1859-66.

Van Oostayen JA, Wasser MN, Griffioen G, van Hogezand RA, Lamers CB, de Roos A. Diagnosis of Crohn's ileitis and monitoring of disease activity: value of Doppler ultrasound of superior mesenteric artery flow. Am J Gastroenterol 1998;93:88-91.

Van Oostayen JA, Wasser MN, van Hogezand RA, et al. Doppler sonography evaluation of superior mesenteric artery flow to assess Crohn's disease activity: correlation with clinical evaluation, Crohn's disease activity index, and alpha 1antitrypsin clearance in feces. Am J Roentgenol 1997;168:429-33.

Van Oostayen JA, Wasser MN, van Hogezand RA, Griffioen G, de Roos A. Activity of Crohn disease assessed by measurement of superior mesenteric artery flow with Doppler US.Radiology 1994;193:551-4.

Wellmann W. Gebel M, Freise J, Grote R. Ultrasound in the diagnosis of ileitis terminalis Crohn. ROFO Fortschr Geb Rontgenstr Nuklearmed 1980;133:146-8.

Wermke W, Gaßmann B. Tumour diagnostics of the liver with echo enhancers. Berlin, Heidelberg, New York: Springer Verlag, 1998: 8-223.

Yekeler E, Danalioglu A, Movasseghi B, et al. Crohn disease activity evaluated by Doppler ultrasonography of the superior mesenteric artery and the affected smallbowel segments. J Ultrasound Med 2005;24:59-65.

Zoli M, Merkel C, Sabba C, Sacerdoti D, Gaiani S, Ferraioli G, Bolondi L. Interobserver and inter-equipment variability of echo-Doppler sonographic evaluation of the superior mesenteric artery. J Ultrasound Med 1996;15:99-106. 


\section{Erklärung}

„Ich, Holger Neye, erkläre, dass ich die vorgelegte Dissertationsschrift mit dem Thema:

PROSPEKTIVER VERGLEICH VON POWER-DOPPLERSONOGRAPHIE UND ILEOKOLOSKOPIE IN DER AKTIVITÄTSBEWERTUNG BEI PATIENTEN MIT MORBUS CROHN selbst verfasst und keine anderen als die angegebenen Quellen und Hilfsmittel benutzt, ohne die (unzulässige) Hilfe Dritter verfasst und auch in Teilen keine Kopien anderer Arbeiten dargestellt habe."

Datum: 09.02.2009 


\section{Lebenslauf}

Mein Lebenslauf wird aus datenschutzrechtlichen Gründen in der elektronischen Version meiner Arbeit nicht veröffentlicht (P romotionsordnung der Medizinischen Fakultät der C harité-Universitätsmedizin Berlin). 